\title{
ANALYSIS OF BDDC ALGORITHMS FOR STOKES PROBLEMS WITH HYBRIDIZABLE DISCONTINUOUS GALERKIN DISCRETIZATIONS*
}

\author{
XUEMIN TU ${ }^{\dagger}$, BIN WANG ${ }^{\ddagger}$, AND JINJIN ZHANG ${ }^{\dagger}$
}

\begin{abstract}
The BDDC (balancing domain decomposition by constraints) methods have been applied to solve the saddle point problem arising from a hybridizable discontinuous Galerkin (HDG) discretization of the incompressible Stokes problem. In the BDDC algorithms, the coarse problem is composed by the edge/face constraints across the subdomain interface for each velocity component. As for the standard approaches of the BDDC algorithms for saddle point problems, these constraints ensure that the BDDC preconditioned conjugate gradient (CG) iterations stay in a subspace where the preconditioned operator is positive definite. However, there are several popular choices of the local stabilization parameters used in the HDG discretizations. Different stabilization parameters change the properties of the resulting discretized operators, and some special observations and tools are needed in the analysis of the condition numbers of the BDDC preconditioned Stokes operators. In this paper, condition number estimates for different choices of stabilization parameters are provided. Numerical experiments confirm the theory.
\end{abstract}

Key words. discontinuous Galerkin, HDG, domain decomposition, BDDC, Stokes problems, Saddle point problems, benign subspace

AMS subject classifications. 65F10, 65N30, 65N55

1. Introduction. Nonoverlapping domain decomposition methods have been studied well for solving saddle-point problems; see, e.g., [9, 10, 16, 18, 19, 20, 25, 26, 27, 28, 29, 35, $36,40]$. In many of these works, the original saddle point problems are reduced to positive definite problems in a subspace called the benign subspace, and the conjugate gradient (CG) method is used to solve the system. In order to keep all CG iterates in the benign subspace, one has to deal with the so-called no-net-flux constraints across subdomain boundaries, which often lead to large coarse-level problems. The no-net-flux constraints can be complicated for the incompressible Stokes equations with a conforming finite element discretization, especially in three dimensions [20]. Moreover, the coarse-level problem will be a bottleneck in large-scale parallel computations, and some inexact solvers in the algorithms are needed to reduce its impact; cf. [8, 13, 14, 30, 31, 32, 33, 40].

The Balancing Domain Decomposition by Constraints (BDDC) algorithms were first introduced for second-order elliptic problems in [7] and analyzed in [22, 23]. Compared to similar nonoverlapping domain decomposition methods, one important feature of the BDDC algorithm is that the Schur complements, arising in the computations, will all be invertible due to the coarse components in the BDDC algorithms, which are given in terms of a set of primal constraints. The BDDC algorithms have been widely applied to different problems such as second-order elliptic problems with mixed and hybrid formulations [28, 29, 40], with isogeometric collocation methods and spectral elements [1,2,4], with staggered discontinuous Galerkin methods [12], with hybridizable discontinuous Galerkin (HDG) discretizations [37], and to the incompressible Stokes problem with a conforming finite element discretization [20] and a weak Galerkin discretization [38], as well as to some nonsymmetric or indefinite problems [17, 34].

The HDG discretization for the incompressible Stokes flow was introduced in [24] and analyzed in [6]. The main features of this approach is that it reduces the globally coupled

\footnotetext{
* Received May 19, 2019. Accepted August 17, 2020. Published online on September 29, 2020. Recommended by Jing Li.

${ }^{\dagger}$ Department of Mathematics, University of Kansas, 1460 Jayhawk Blvd, Lawrence, KS 66045-7594, U.S.A. ( $\{$ xuemin, jinjinzhang $\} @ k u . e d u$ ).

${ }^{\ddagger}$ Department of Mathematical Sciences, Hood College, 401 Roesmont Ave., Frederick, MD 21701, U.S.A. (wang@hood.edu).
} 
unknowns to the numerical trace of the velocity on the element boundaries and the mean of the pressure on the element. The size of the reduced saddle point problem is significantly smaller compared to the original one. In [24], the reduced saddle point problem is solved by an augmented Lagrange approach. An additional time-dependent problem is introduced and solved by a backward-Euler method. Here, we solve the reduced saddle point problem directly using BDDC methods. Similarly to earlier domain decomposition works on saddle point problems [16, 20, 26, 28, 38], we reduce the saddle point problem to positive definite problems in a benign subspace, and therefore the CG method can be used to solve the resulting system. Due to the discontinuous pressure basis functions in this HDG discretization, the complicated no-net-flux condition, which is needed to make sure that all CG iterates stay in the benign subspace, can be enforced by edge and face average constraints for each velocity component in two and three dimensions, respectively. These required constraints are the same as those for the elliptic problems with the HDG discretizations [37] and the Stokes problems with weak Galerkin discretization [38].

Usually, the analysis of the BDDC algorithm for the Stokes problem consists of several components as in [20, 38]. First, we need to prove the inf-sup stability of the HDG methods. Based on this result, we establish the relation between the Stokes and harmonic extensions. Finally, combining these results and the condition number bound for the elliptic problem in [37], we obtain the condition number estimate for the BDDC-preconditioned Stokes operator. However, the relation between the Stokes and harmonic extensions can be established only for one special choice of the local stabilization parameter $\tau_{\kappa}$, which was used in [38]. Therefore, a similar analysis cannot be used for other popular choices of $\tau_{\kappa}$ in [24]. In this paper, we provide a complete condition number estimate for the different choices of $\tau_{\kappa}$ in [24]. The important observation is that different choices of $\tau_{\kappa}$ do not change the divergence-free space of the Stokes equation. They only change the coefficient of one nonnegative component in the corresponding norms. Based on these facts, we can estimate the condition number for other choices of $\tau_{\kappa}$ using the result for the special choice, which can be proved as in [38].

The remainder of the paper is organized as follows. The HDG discretization for the Stokes problem is described in Section 2. In Section 3, the original system is reduced to an interface problem. A BDDC preconditioner is then formulated in Section 4, and the benign subspaces, where the preconditioned operator is positive definite, are introduced. Some auxiliary results are given in Section 5, and the condition number estimate for the system with the BDDC preconditioner is provided in Section 6. Finally, we give some computational results in Section 7 to confirm our theory.

2. A Stokes problem and the HDG discretization. The following Stokes problem is defined on a bounded polygonal domain $\Omega$, in two or three dimensions, with a Dirichlet boundary condition:

$$
\left\{\begin{aligned}
-\triangle \mathbf{u}+\nabla p & =\mathbf{f}, & & \text { in } \Omega, \\
\nabla \cdot \mathbf{u} & =0, & & \text { in } \Omega, \\
\mathbf{u} & =g, & & \text { on } \partial \Omega,
\end{aligned}\right.
$$

where $\mathbf{f} \in L^{2}(\Omega)$ and $g \in H^{1 / 2}(\partial \Omega)$. Here we assume that $g=0$ without loss of generality. The solution of (2.1) is unique up to an additive constant for the pressure $p$. Here we will look for the solution with the pressure $p$ having a zero average over the domain $\Omega$. 
We follow the approach in $[6,24]$ and introduce the HDG method for the Stokes equation defined in (2.1) as follows:

$$
\left\{\begin{array}{rlrl}
\mathbf{L}-\nabla \mathbf{u}=0, & & \text { in } \Omega, \\
-\nabla \cdot \mathbf{L}+\nabla p=\mathbf{f}, & & \text { in } \Omega, \\
\nabla \cdot \mathbf{u}=0, & & \text { in } \Omega, \\
\mathbf{u} & =0, & & \text { in } \partial \Omega .
\end{array}\right.
$$

Let $P_{k}(D)$ be the space of polynomials of order at most $k$ on $D$. We define the spaces $\mathbf{P}_{k}(D)=\left[P_{k}(D)\right]^{n}$ (with $n=2$ and 3 for two and three dimensions, respectively) and $\mathcal{P}_{k}(D)=\left[P_{k}(D)\right]^{n \times n}$. L, $\mathbf{u}$, and $p$ will be approximated by these discontinuous finite element spaces defined on a shape-regular and quasi-uniform triangulation of $\Omega$ denoted by $\mathcal{T}_{h}$. Let $h$ be the characteristic element size of $\mathcal{T}_{h}$ and $\kappa$ be an element in $\mathcal{T}_{h}$. The union of the faces of all the elements $\kappa$ is denoted by $\mathcal{E}$. $\mathcal{E}_{i}$ and $\mathcal{E}_{\partial}$ are two subsets of $\mathcal{E}$ indicating the faces in the domain interior and on the domain boundary, respectively.

For each element $\kappa$, we denote by $(\cdot, \cdot)_{\kappa}$ and $\langle\cdot, \cdot\rangle_{\partial \kappa}$ the $L^{2}$-inner products of functions or vector-valued functions on $\kappa$ and $\partial \kappa$, respectively. For all $\kappa \in \mathcal{T}_{h}$, we want to find $\left(\mathbf{L}_{h}, \mathbf{u}_{h}, p_{h}\right) \in\left(\mathcal{P}_{k}(\kappa), \mathbf{P}_{k}(\kappa), P_{k}(\kappa)\right)$ such that

$$
\left\{\begin{aligned}
-\left(\mathbf{L}_{h}, \mathbf{G}_{h}\right)_{\kappa}-\left(\mathbf{u}_{h}, \nabla \cdot \mathbf{G}_{h}\right)_{\kappa}+\left\langle\widehat{\mathbf{u}}_{h}, \mathbf{G}_{h} \mathbf{n}\right\rangle_{\partial \kappa} & =0, & & \forall \mathbf{G}_{h} \in \mathcal{P}_{k}(\kappa), \\
\left(\mathbf{L}_{h}, \nabla \mathbf{v}_{h}\right)_{\kappa}-\left(p_{h}, \nabla \cdot \mathbf{v}_{h}\right)_{\kappa}-\left\langle\widehat{\mathbf{L}}_{h} \mathbf{n}-\widehat{p}_{h} \mathbf{n}, \mathbf{v}_{h}\right\rangle_{\partial \kappa} & =\left(\mathbf{f}, \mathbf{v}_{h}\right)_{\kappa}, & & \forall \mathbf{v}_{h} \in \mathbf{P}_{k}(\kappa), \\
\left(\mathbf{u}_{h}, \nabla q_{h}\right)_{\kappa}-\left\langle\widehat{\mathbf{u}}_{h} \cdot \mathbf{n}, q_{h}\right\rangle_{\partial \kappa} & =0, & & \forall q_{h} \in P_{k}(\kappa),
\end{aligned}\right.
$$

where $\widehat{\mathbf{L}}_{h}, \widehat{\mathbf{u}}_{h}$, and $\widehat{p}_{h}$ are the numerical traces that approximate $\mathbf{L}_{h}, \mathbf{u}_{h}$, and $p_{h}$ on $\partial \kappa$, respectively.

Define the following finite element spaces:

$$
\begin{aligned}
\mathbf{G}_{k} & =\left\{\mathbf{G}_{h} \in\left[L^{2}(\Omega)\right]^{n \times n}:\left.\mathbf{G}_{h}\right|_{\kappa} \in \mathcal{P}_{k}(\kappa) \quad \forall \kappa \in \Omega\right\}, \\
\mathbf{V}_{k} & =\left\{\mathbf{v}_{h} \in\left[L^{2}(\Omega)\right]^{n}:\left.\mathbf{v}_{h}\right|_{\kappa} \in \mathbf{P}_{k}(\kappa) \quad \forall \kappa \in \Omega\right\}, \\
W_{k} & =\left\{p_{h} \in L^{2}(\Omega):\left.p_{h}\right|_{\kappa} \in P_{k}(\kappa), \quad \int_{\Omega} p_{h}=0, \quad \forall \kappa \in \Omega\right\}, \\
\mathbf{M}_{k} & =\left\{\boldsymbol{\mu}_{h} \in\left[L^{2}(\mathcal{E})\right]^{n}:\left.\boldsymbol{\mu}_{h}\right|_{e} \in \mathbf{P}_{k}(e) \quad \forall e \in \mathcal{E}\right\} .
\end{aligned}
$$

Let $\boldsymbol{\Lambda}_{k}=\left\{\boldsymbol{\mu}_{h} \in \mathbf{M}_{k}:\left.\boldsymbol{\mu}_{h}\right|_{e}=0 \forall e \in \partial \Omega\right\}$. To simplify notation, the subscript $k$ will be omitted from now on.

The numerical trace $\widehat{\mathbf{u}}_{h}$ is defined as $\boldsymbol{\lambda}_{h}$, where $\boldsymbol{\lambda}_{h} \in \boldsymbol{\Lambda}$. In this paper, the following numerical flux is considered:

$$
\widehat{\mathbf{L}}_{h} \mathbf{n}-\widehat{p}_{h} \mathbf{n}=\mathbf{L}_{h} \mathbf{n}-p_{h} \mathbf{n}-\tau_{\kappa}\left(\mathbf{u}_{h}-\boldsymbol{\lambda}_{h}\right) \quad \text { on } \partial \kappa,
$$

where $\tau_{\kappa}$ is a local stabilization parameter; see $[6,24]$ for a more general discussion.

Plugging in the definitions of the numerical trace $\widehat{\mathbf{u}}_{h}=\lambda_{h}$ and the numerical flux $\widehat{\mathbf{L}}_{h} \mathbf{n}-\widehat{p}_{h} \mathbf{n}$ in (2.2) and summing over all elements, we can write the discrete problem resulting from an HDG discretization as: find $\left(\mathbf{L}_{h}, \mathbf{u}_{h}, p_{h}, \boldsymbol{\lambda}_{h}\right) \in(\mathbf{G}, \mathbf{V}, W, \boldsymbol{\Lambda})$ such that for all $\left(\mathbf{G}_{h}, \boldsymbol{v}_{h}, q_{h}, \boldsymbol{\mu}_{h}\right) \in(\mathbf{G}, \mathbf{V}, W, \boldsymbol{\Lambda})$,

$$
\left\{\begin{aligned}
-\left(\mathbf{L}_{h}, \mathbf{G}_{h}\right)_{\mathcal{T}_{h}}-\left(\mathbf{u}_{h}, \nabla \cdot \mathbf{G}_{h}\right)_{\mathcal{T}_{h}}+\left\langle\boldsymbol{\lambda}_{h}, \mathbf{G}_{h} \mathbf{n}\right\rangle_{\partial \mathcal{T}_{h}} & =0 \\
\left(\mathbf{L}_{h}, \nabla \mathbf{v}_{h}\right)_{\mathcal{T}_{h}}-\left(p_{h}, \nabla \cdot \mathbf{v}_{h}\right)_{\mathcal{T}_{h}}-\left\langle\mathbf{L}_{h} \mathbf{n}-p_{h} \mathbf{n}-\tau_{\kappa}\left(\mathbf{u}_{h}-\boldsymbol{\lambda}_{h}\right), \mathbf{v}_{h}\right\rangle_{\partial \mathcal{T}_{h}} & =\left(\mathbf{f}, \mathbf{v}_{h}\right)_{\mathcal{T}_{h}} \\
\left(\mathbf{u}_{h}, \nabla q_{h}\right)_{\mathcal{T}_{h}}-\left\langle\boldsymbol{\lambda}_{h} \cdot \mathbf{n}, q_{h}\right\rangle_{\partial \mathcal{T}_{h}} & =0 \\
\left\langle\mathbf{L}_{h} \mathbf{n}-p_{h} \mathbf{n}-\tau_{\kappa}\left(\mathbf{u}_{h}-\boldsymbol{\lambda}_{h}\right), \boldsymbol{\mu}_{h}\right\rangle_{\partial \mathcal{T}_{h}} & =0
\end{aligned}\right.
$$


where $(\cdot, \cdot)_{\mathcal{T}_{h}}=\sum_{\kappa \in \mathcal{T}_{h}}(\cdot, \cdot)_{\kappa}$ and $\langle\cdot, \cdot\rangle_{\partial \mathcal{T}_{h}}=\sum_{\kappa \in \mathcal{T}_{h}}\langle\cdot, \cdot\rangle_{\partial \kappa}$, respectively.

In order to obtain the matrix form of (2.3), we introduce the following operators as in [5],

$$
\begin{array}{llll}
A_{\mathbf{L L}}: \mathbf{G} \rightarrow \mathbf{G}, & A_{\mathbf{u L}}: \mathbf{G} \rightarrow \mathbf{V}, & A_{\boldsymbol{\lambda} \mathbf{L}}: \mathbf{G} \rightarrow \boldsymbol{\Lambda}, & A_{\mathbf{u u}}: \mathbf{V} \rightarrow \mathbf{V}, \\
A_{\boldsymbol{\lambda} \mathbf{u}}: \mathbf{V} \rightarrow \boldsymbol{\Lambda}, & B_{p \mathbf{u}}: \mathbf{V} \rightarrow W, & A_{\boldsymbol{\lambda} \boldsymbol{\lambda}}: \boldsymbol{\Lambda} \rightarrow \boldsymbol{\Lambda}, & B_{p \boldsymbol{\lambda}}: \boldsymbol{\Lambda} \rightarrow W,
\end{array}
$$

defined as

$$
\begin{array}{rlrl}
\left(A_{\mathbf{L L}} \mathbf{L}_{h}, \mathbf{G}_{h}\right) & =-\left(\mathbf{L}_{h}, \mathbf{G}_{h}\right)_{\mathcal{T}_{h}}, & & \left(A_{\mathbf{u L}} \mathbf{L}_{h}, \mathbf{v}_{h}\right)=-\left(\nabla \cdot \mathbf{L}_{h}, \mathbf{v}_{h}\right)_{\mathcal{T}_{h}}, \\
\left(A_{\boldsymbol{\lambda} \mathbf{L}} \mathbf{L}_{h}, \boldsymbol{\mu}_{h}\right)=\left\langle\mathbf{L}_{h} \mathbf{n}, \boldsymbol{\mu}_{h}\right\rangle_{\partial \mathcal{T}_{h}}, & & \left(A_{\mathbf{u u}} \mathbf{u}_{h}, \mathbf{v}_{h}\right)=\tau_{\kappa}\left\langle\mathbf{u}_{h}, \mathbf{v}_{h}\right\rangle_{\partial \mathcal{T}_{h}}, \\
\left(A_{\boldsymbol{\lambda} \mathbf{u}} \mathbf{u}_{h}, \boldsymbol{\mu}_{h}\right) & =-\tau_{\kappa}<\mathbf{u}_{h}, \boldsymbol{\mu}_{h}>_{\partial \mathcal{T}_{h}}, & & \left(A_{\boldsymbol{\lambda} \boldsymbol{\lambda}} \boldsymbol{\lambda}_{h}, \boldsymbol{\mu}_{h}\right)=\tau_{\kappa}<\boldsymbol{\lambda}_{h}, \boldsymbol{\mu}_{h}>_{\partial \mathcal{T}_{h}} \\
\left(B_{p \mathbf{u}} \mathbf{v}_{h}, p_{h}\right) & =\left(\mathbf{v}_{h}, \nabla p_{h}\right)_{\mathcal{T}_{h}}, & & \left(B_{p \boldsymbol{\lambda}} \boldsymbol{\lambda}_{h}, p_{h}\right)=-<\boldsymbol{\lambda}_{h} \cdot \boldsymbol{n}, p_{h}>_{\partial \mathcal{T}_{h}},
\end{array}
$$

for all $\mathbf{L}_{h}, \mathbf{G}_{h} \in \mathbf{G}, \mathbf{u}_{h}, \mathbf{v}_{h} \in \mathbf{V}, p_{h}, q_{h} \in W$, and $\boldsymbol{\lambda}, \boldsymbol{\mu} \in \mathbf{\Lambda}$.

Equation (2.3) can thus be rewritten in matrix form as

$$
\left[\begin{array}{cccc}
A_{\mathbf{L L}} & A_{\mathbf{u L}}^{T} & A_{\lambda \mathbf{L}}^{T} & 0 \\
A_{\mathbf{u L}} & A_{\mathbf{u u}} & A_{\lambda \mathbf{u}}^{T} & B_{p \mathbf{u}}^{T} \\
A_{\boldsymbol{\lambda} \mathbf{L}} & A_{\boldsymbol{\lambda u}} & A_{\boldsymbol{\lambda} \boldsymbol{\lambda}} & B_{p \boldsymbol{\lambda}}^{T} \\
0 & B_{p \mathbf{u}} & B_{p \boldsymbol{\lambda}} & 0
\end{array}\right]\left[\begin{array}{c}
\mathbf{L} \\
\mathbf{u} \\
\boldsymbol{\lambda} \\
p
\end{array}\right]=\left[\begin{array}{c}
\mathbf{0} \\
\mathbf{F}_{h} \\
\mathbf{0} \\
0
\end{array}\right]
$$

where $\mathbf{F}_{h}=\left(\mathbf{f}, \mathbf{v}_{h}\right)_{\mathcal{T}_{h}}$ and $\mathbf{L}, \mathbf{u}, \boldsymbol{\lambda}$, and $p$ denote the unknowns associated with $\mathbf{L}_{h}, \mathbf{u}_{h}, \boldsymbol{\lambda}_{h}$, and $p_{h}$, respectively.

Let

$$
A_{a}=\left[\begin{array}{ccc}
A_{\mathbf{L L}} & A_{\mathbf{u L}}^{T} & A_{\lambda \mathbf{L}}^{T} \\
A_{\mathbf{u L}} & A_{\mathbf{u u}} & A_{\lambda \mathbf{u}}^{T} \\
A_{\boldsymbol{\lambda} \mathbf{L}} & A_{\boldsymbol{\lambda} \mathbf{u}} & A_{\boldsymbol{\lambda} \boldsymbol{\lambda}}
\end{array}\right], \quad B_{a}^{T}=\left[\begin{array}{c}
0 \\
B_{p \mathbf{u}}^{T} \\
B_{p \boldsymbol{\lambda}}^{T}
\end{array}\right], \quad u_{a}=\left[\begin{array}{c}
\mathbf{L} \\
\mathbf{u} \\
\lambda
\end{array}\right], \quad \text { and } \mathbf{F}_{a}=\left[\begin{array}{c}
\mathbf{0} \\
\mathbf{F}_{h} \\
\mathbf{0}
\end{array}\right]
$$

Then, the global problem (2.5) can be rewritten as the saddle point problem

$$
\left[\begin{array}{cc}
A_{a} & B_{a}^{T} \\
B_{a} & 0
\end{array}\right]\left[\begin{array}{c}
u_{a} \\
p
\end{array}\right]=\left[\begin{array}{c}
\mathbf{F}_{a} \\
0
\end{array}\right]
$$

where $A_{a}$ is the same as the matrix obtained using an HDG discretization for the elliptic problem as discussed in [37].

For each $\kappa$, we decompose the pressure degrees of freedom $p$ into the element average pressure $p_{0 e}$ and the rest, called the element interior pressure $p_{i}$, and we let $W=W_{i} \oplus W_{0 e}$, correspondingly. We can rewrite (2.5) as

$$
\left[\begin{array}{ccccc}
A_{\mathbf{L L}} & A_{\mathbf{u L}}^{T} & 0 & A_{\boldsymbol{\lambda} \mathbf{L}}^{T} & 0 \\
A_{\mathbf{u L}} & A_{\mathbf{u u}} & B_{p_{i} \mathbf{u}}^{T} & A_{\boldsymbol{\lambda} \mathbf{u}}^{T} & 0 \\
0 & B_{p_{i} \mathbf{u}} & 0 & B_{p_{i} \boldsymbol{\lambda}} & 0 \\
A_{\boldsymbol{\lambda} \mathbf{L}} & A_{\boldsymbol{\lambda u}} & B_{p_{i \boldsymbol{\lambda}}}^{T} & A_{\boldsymbol{\lambda} \boldsymbol{\lambda}} & B_{p_{0 e} \boldsymbol{\lambda}}^{T} \\
0 & 0 & 0 & B_{p_{0 e} \boldsymbol{\lambda}} & 0
\end{array}\right]\left[\begin{array}{c}
\mathbf{L} \\
\mathbf{u} \\
p_{i} \\
\boldsymbol{\lambda} \\
p_{0 e}
\end{array}\right]=\left[\begin{array}{c}
\mathbf{0} \\
\mathbf{F}_{h} \\
0 \\
\mathbf{0} \\
0
\end{array}\right]
$$

Here the matrix

$$
\left[\begin{array}{ccc}
A_{\mathbf{L L}} & A_{\mathbf{u L}}^{T} & 0 \\
A_{\mathbf{u L}} & A_{\mathbf{u u}} & B_{p_{i} \mathbf{u}}^{T} \\
0 & B_{p_{i} \mathbf{u}} & 0
\end{array}\right]
$$


is nonsingular and block diagonal with each block corresponding to one element $\kappa$. Therefore, $\mathbf{L}, \mathbf{u}$, and $p_{i}$ can be eliminated in each element independently from (2.7), and a system for $\boldsymbol{\lambda}$ and $p_{0 e}$ only is obtained:

$$
\left[\begin{array}{cc}
A & B^{T} \\
B & 0
\end{array}\right]\left[\begin{array}{c}
\lambda \\
p_{0 e}
\end{array}\right]=\left[\begin{array}{l}
b \\
0
\end{array}\right]
$$

where

$$
A=A_{\boldsymbol{\lambda} \mathbf{\lambda}}-\left[\begin{array}{lll}
A_{\boldsymbol{\lambda} \mathbf{L}} & A_{\boldsymbol{\lambda} \mathbf{u}} & B_{p_{i} \mathbf{\lambda}}^{T}
\end{array}\right]\left[\begin{array}{ccc}
A_{\mathbf{L L}} & A_{\mathbf{u L}}^{T} & 0 \\
A_{\mathbf{u L}} & A_{\mathbf{u u}} & B_{p_{i} \mathbf{u}}^{T} \\
0 & B_{p_{i}, \mathbf{u}} & 0
\end{array}\right]^{-1}\left[\begin{array}{c}
A_{\lambda \mathbf{L}}^{T} \\
A_{\boldsymbol{\lambda} \mathbf{u}}^{T} \\
B_{p_{i} \boldsymbol{\lambda}}
\end{array}\right],
$$

and

$$
B=B_{p_{0 e} \boldsymbol{\lambda}}, \quad b=-\left[\begin{array}{lll}
A_{\boldsymbol{\lambda} \mathbf{L}} & A_{\boldsymbol{\lambda} \mathbf{u}} & B_{p_{i} \boldsymbol{\lambda}}^{T}
\end{array}\right]\left[\begin{array}{ccc}
A_{\mathbf{L L}} & A_{\mathbf{u L}}^{T} & 0 \\
A_{\mathbf{u L}} & A_{\mathbf{u u}} & B_{p_{i} \mathbf{u}}^{T} \\
0 & B_{p_{i}, \mathbf{u}} & 0
\end{array}\right]^{-1}\left[\begin{array}{c}
\mathbf{0} \\
F_{h} \\
0
\end{array}\right]
$$

After solving for $\boldsymbol{\lambda}$ and $p_{0 e}$ using (2.8), given the value of $\boldsymbol{\lambda}$ on $\partial \kappa$, the unknowns $\mathbf{L}, \mathbf{u}, p_{i}$ are uniquely determined on each element $\kappa$, and the solution of (2.7) can be completed.

The system (2.8) is the matrix form of the following problem: find $\boldsymbol{\lambda} \in \boldsymbol{\Lambda}$ and $p_{0 e} \in W_{0 e}$ such that

$$
\left\{\begin{aligned}
a_{h}(\boldsymbol{\lambda}, \boldsymbol{\mu})+b_{h}\left(p_{0 e}, \boldsymbol{\mu}\right) & =l_{h}(\boldsymbol{\mu}), & & \forall \boldsymbol{\mu} \in \boldsymbol{\Lambda}, \\
b_{h}\left(q_{0 e}, \boldsymbol{\lambda}\right) & =0, & & \forall q_{0 e} \in W_{0 e} .
\end{aligned}\right.
$$

Here,

$$
\begin{aligned}
a_{h}(\boldsymbol{\eta}, \boldsymbol{\mu}) & =\sum_{\kappa \in \mathcal{T}_{h}}(\mathcal{L}(\boldsymbol{\eta}), \mathcal{L}(\boldsymbol{\mu}))_{\kappa}+\left\langle\tau_{\kappa}(\mathcal{U}(\boldsymbol{\eta})-\boldsymbol{\eta}),(\mathcal{U}(\boldsymbol{\mu})-\boldsymbol{\mu})\right\rangle_{\partial \kappa}, \\
b_{h}\left(p_{0 e}, \boldsymbol{\mu}\right) & =-\left\langle p_{0 e}, \boldsymbol{\mu} \cdot \mathbf{n}\right\rangle_{\partial \mathcal{T}_{h}} \\
l_{h}(\boldsymbol{\mu}) & =\sum_{\kappa \in \mathcal{T}_{h}} l_{\kappa}(\boldsymbol{\eta}, \boldsymbol{\mu})=\sum_{\kappa \in \mathcal{T}_{h}}\left(f_{h}, \mathcal{U}(\boldsymbol{\mu})\right)_{\kappa},
\end{aligned}
$$

where $\mathcal{L}(\boldsymbol{\mu})$ and $\mathcal{U}(\boldsymbol{\mu})$ are the unique solution $\left(\mathbf{L}_{h}=\mathcal{L}(\boldsymbol{\mu}), \mathbf{u}_{h}=\mathcal{U}(\boldsymbol{\mu})\right)$ of the local element problem (2.7) with $\boldsymbol{\lambda}=\boldsymbol{\mu}$ and $\mathbf{F}_{h}=0$; see [24, Theorem 2.1] for details.

In next two sections, following the same procedures as in [38], we will use a BDDC algorithm to solve the system in (2.8) for $\lambda$ and $p_{0 e}$.

3. Reduced subdomain interface problem. The domain $\Omega$ is decomposed into $N$ nonoverlapping subdomains $\Omega_{i}$ with diameters $H_{i}, i=1, \ldots, N$. Let $H=\max _{i} H_{i}$. Each subdomain is assumed to be a union of shape-regular coarse triangles, and the number of such elements forming an individual subdomain are assumed uniformly bounded. The open sets shared by two subdomains are defined as faces. We define $\Gamma$ as the interface between the subdomains. Let $\Gamma_{h}:=\left(\cup_{i \neq j} \partial \Omega_{i, h} \cap \partial \Omega_{j, h}\right) \backslash \partial \Omega_{h}$ be the set of the interface nodes. Here, $\partial \Omega_{i, h}$ is the set of nodes on $\partial \Omega_{i}$, and $\partial \Omega_{h}$ is that of $\partial \Omega$. The triangulation of each subdomain is assumed to be quasi-uniform.

Let $\widehat{\Lambda}_{\Gamma}$ be the space containing the degrees of freedom associated with $\Gamma, \Lambda_{I}^{(i)}$ be subdomain interior velocity numerical trace spaces, and $W_{I}^{(i)}$ be subdomain interior pressure spaces. By these definitions, we can see that the elements of $\Lambda_{I}^{(i)}$ are supported in the subdomain $\Omega_{i}$ 
and vanish on $\partial \Omega_{i}$. The elements of $W_{I}^{(i)}$ are restrictions of the pressure variables to $\Omega_{i}$ with $\int_{\Omega_{i}} p_{I}^{(i)}=0$.

We define

$$
\boldsymbol{\Lambda}_{I}=\prod_{i=1}^{N} \boldsymbol{\Lambda}_{I}^{(i)}, \quad W_{I}=\prod_{i=1}^{N} W_{I}^{(i)} .
$$

The numerical trace of the velocity $\Lambda$ and the element average pressure $W_{0 e}$ can be decomposed into:

$$
\boldsymbol{\Lambda}=\boldsymbol{\Lambda}_{I} \oplus \widehat{\boldsymbol{\Lambda}}_{\Gamma}, \quad W_{0 e}=W_{I} \oplus W_{0} .
$$

Here $W_{0}$ is a subspace of $W$. The elements of $W_{0}$ have constant values $p_{0}^{(i)}$ in each subdomain $\Omega_{i}$, and $\sum_{i=1}^{N} p_{0}^{(i)} m\left(\Omega_{i}\right)=0$, where $m\left(\Omega_{i}\right)$ is the measure of the subdomain $\Omega_{i}$.

Let $\Lambda_{\Gamma}^{(i)}$ be the space of interface velocity numerical trace variables of the subdomain $\Omega_{i}$ and $\Lambda_{\Gamma}=\prod_{i=1}^{N} \Lambda_{\Gamma}^{(i)}$. We note that generally the functions in $\Lambda_{\Gamma}$ are discontinuous across the interface. The restriction operators $R_{\Gamma}^{(i)}: \widehat{\Lambda}_{\Gamma} \rightarrow \Lambda_{\Gamma}^{(i)}$ map functions in the continuous global interface velocity numerical trace variable space $\widehat{\Lambda}_{\Gamma}$ to the subdomain component space $\Lambda_{\Gamma}^{(i)}$. The operator $R_{\Gamma}$ is defined as the direct sum of $R_{\Gamma}^{(i)}$.

The global problem (2.8) can be written as

$$
\left[\begin{array}{cccc}
A_{I I} & B_{I I}^{T} & A_{\Gamma I}^{T} & 0 \\
B_{I I} & 0 & B_{I \Gamma} & 0 \\
A_{\Gamma I} & B_{I \Gamma}^{T} & A_{\Gamma \Gamma} & B_{0 \Gamma}^{T} \\
0 & 0 & B_{0 \Gamma} & 0
\end{array}\right]\left[\begin{array}{c}
\lambda_{I} \\
p_{I} \\
\lambda_{\Gamma} \\
p_{0}
\end{array}\right]=\left[\begin{array}{c}
b_{I} \\
0 \\
b_{\Gamma} \\
0
\end{array}\right],
$$

and it is assembled from the subdomain problems

$$
\left[\begin{array}{cccc}
A_{I I}^{(i)} & B_{I I}^{(i)^{T}} & A_{\Gamma I}^{(i)^{T}} & 0 \\
B_{I I}^{(i)} & 0 & B_{I \Gamma}^{(i)} & 0 \\
A_{\Gamma I}^{(i)} & B_{I \Gamma}^{(i)^{T}} & A_{\Gamma \Gamma}^{(i)} & B_{0 \Gamma}^{(i)^{T}} \\
0 & 0 & B_{0 \Gamma}^{(i)} & 0
\end{array}\right]\left[\begin{array}{c}
\lambda_{I}^{(i)} \\
p_{I}^{(i)} \\
\lambda_{\Gamma}^{(i)} \\
p_{0}^{(i)}
\end{array}\right]=\left[\begin{array}{c}
b_{I}^{(i)} \\
0 \\
b_{\Gamma}^{(i)} \\
0
\end{array}\right] .
$$

We can eliminate the subdomain interior variables $\boldsymbol{\lambda}_{I}^{(i)}$ and $p_{I}^{(i)}$ in each subdomain independently and define the subdomain Schur complement $S_{\Gamma}^{(i)}$ as follows: given $\lambda_{\Gamma}^{(i)} \in \Lambda_{\Gamma}^{(i)}$, determine $S_{\Gamma}^{(i)} \boldsymbol{\lambda}_{\Gamma}^{(i)}$ such that

$$
\left[\begin{array}{ccc}
A_{I I}^{(i)} & B_{I I}^{(i) T} & A_{\Gamma I}^{(i) T} \\
B_{I I}^{(i)} & 0 & B_{\Gamma I}^{(i) T} \\
A_{\Gamma I}^{(i)} & B_{\Gamma I}^{(i)} & A_{\Gamma \Gamma}^{(i)}
\end{array}\right]\left[\begin{array}{c}
\lambda_{I}^{(i)} \\
p_{I}^{(i)} \\
\lambda_{\Gamma}^{(i)}
\end{array}\right]=\left[\begin{array}{c}
\mathbf{0} \\
0 \\
S_{\Gamma}^{(i)} \lambda_{\Gamma}^{(i)}
\end{array}\right] .
$$

The global interface problem is assembled from the subdomain interface problems and can be written as: find $\left(\boldsymbol{\lambda}_{\Gamma}, p_{0}\right) \in\left(\widehat{\Lambda}_{\Gamma}, W_{0}\right)$ such that

$$
\widehat{S}\left[\begin{array}{c}
\lambda_{\Gamma} \\
p_{0}
\end{array}\right]=\left[\begin{array}{c}
g_{\Gamma} \\
0
\end{array}\right], \quad \text { where } \quad \widehat{S}=\left[\begin{array}{cc}
\widehat{S}_{\Gamma} & \widehat{B}_{0 \Gamma}^{T} \\
\widehat{B}_{0 \Gamma} & 0
\end{array}\right],
$$




$$
\begin{gathered}
\widehat{S}_{\Gamma}=\sum_{i=1}^{N} R_{\Gamma}^{(i) T} S_{\Gamma}^{(i)} R_{\Gamma}^{(i)}, \quad \widehat{B}_{0 \Gamma}=\sum_{i=1}^{N} B_{0 \Gamma}^{(i)} R_{\Gamma}^{(i)}, \quad \text { and } \\
g_{\Gamma}=\sum_{i=1}^{N} R_{\Gamma}^{(i) T}\left\{b_{\Gamma}^{(i)}-\left[\begin{array}{ll}
A_{\Gamma I}^{(i)} & B_{I \Gamma}^{(i) T}
\end{array}\right]\left[\begin{array}{cc}
A_{I I}^{(i)} & B_{I I}^{(i) T} \\
B_{I I}^{(i)} & 0
\end{array}\right]^{-1}\left[\begin{array}{c}
b_{I}^{(i)} \\
0
\end{array}\right]\right\} .
\end{gathered}
$$

By these definitions, the operator $\widehat{S}$ is symmetric indefinite, while $\widehat{S}_{\Gamma}$ is symmetric positive definite. In the next section, we will introduce a BDDC preconditioner and show that the BDDC preconditioned operator is symmetric positive definite in a subspace.

4. The BDDC preconditioner. In order to introduce the BDDC preconditioner, we further decompose the subdomain interface velocity variables into the primal and dual interface velocity variables. We retain continuity of the primal velocity variables, but relax that of the dual velocity variables across the subdomain interface. The primal interface velocity space is denoted by $\widehat{\Lambda}_{\Pi}$, and the dual space $\Lambda_{\triangle}$ is the direct sum of the $\Lambda_{\triangle}^{(i)}$, which are spanned by the remaining interface degrees of freedom (after the primal variables) in each subdomain. A partially assembled interface space $\widetilde{\boldsymbol{\Lambda}}_{\Gamma}$ can be defined as

$$
\widetilde{\Lambda}_{\Gamma}=\widehat{\Lambda}_{\Pi} \oplus \boldsymbol{\Lambda}_{\Delta}=\widehat{\Lambda}_{\Pi} \oplus \prod_{i=1}^{N} \boldsymbol{\Lambda}_{\Delta}^{(i)} .
$$

We note that $\widehat{\Lambda}_{\Pi}$ is the coarse-level velocity space, which makes all the linear systems nonsingular.

Moreover, several restriction, extension, and scaling operators between different spaces have to be defined. The restriction operator $\bar{R}_{\Gamma}^{(i)}$ restricts functions in the space $\widetilde{\Lambda}_{\Gamma}$ to the components $\Lambda_{\Gamma}^{(i)}$ of the subdomain $\Omega_{i}$, and the direct sum of $\bar{R}_{\Gamma}^{(i)}$ is denoted by $\bar{R}_{\Gamma}$. The restriction operator $R_{\Delta}^{(i)}$ maps the functions from $\widehat{\Lambda}_{\Gamma}$ to $\Lambda_{\Delta}^{(i)}$, while $R_{\Gamma \Pi}$ is a restriction operator from $\widehat{\boldsymbol{\Lambda}}_{\Gamma}$ to its subspace $\widehat{\boldsymbol{\Lambda}}_{\Pi}$. The direct sum of $R_{\Gamma \Pi}$ and $R_{\Delta}^{(i)}$ is denoted by $\widetilde{R}_{\Gamma}$.

Given a point $x$ on the subdomain interface, let $\mathcal{I}_{x}$ be the set of indices of the subdomains that have $x$ on their boundaries, and card $\left(\mathcal{I}_{x}\right)$ gives the number of subdomain boundaries to which $x$ belongs. A positive scaling factor $\delta_{i}^{\dagger}(x)$ is defined as follows:

$$
\delta_{i}^{\dagger}(x)=\frac{1}{\operatorname{card}\left(\mathcal{I}_{x}\right)}, \quad x \in \partial \Omega_{i, h} \cap \Gamma_{h} .
$$

On each face, $\delta_{i}^{\dagger}(x)$ is constant. $R_{D, \Delta}^{(i)}$ is obtained by multiplying each row of $R_{\Delta}^{(i)}$ by the scaling factor. The direct sum of $R_{\Gamma \Pi}$ and $R_{D, \Delta}^{(i)}$ gives us the scaled operators $\widetilde{R}_{D, \Gamma}$.

$S_{\Gamma}$ denotes the direct sum of $S_{\Gamma}^{(i)}$, defined in (3.1). A partially assembled interface velocity Schur complement is defined by $\widetilde{S}_{\Gamma}=\bar{R}_{\Gamma}^{T} S_{\Gamma} \bar{R}_{\Gamma}$. The operator $\widetilde{B}_{0 \Gamma}$ maps the partially assembled interface velocity space $\widetilde{\Lambda}_{\Gamma}$ into the space of right-hand sides corresponding to $W_{0}$ and can be written as $\widetilde{B}_{0 \Gamma}=\sum_{i=1}^{N} B_{0 \Gamma}^{(i)} \bar{R}_{\Gamma}^{(i)}$.

Let

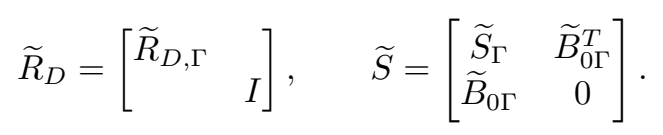


Then the preconditioner for solving the global interface Stokes problem is

$$
M^{-1}=\widetilde{R}_{D}^{T} \widetilde{S}^{-1} \widetilde{R}_{D}
$$

and the preconditioned BDDC algorithm is then of the form: find $\left(\boldsymbol{\lambda}_{\Gamma}, p_{0}\right) \in\left(\widehat{\Lambda}_{\Gamma}, W_{0}\right)$ such that

$$
\widetilde{R}_{D}^{T} \widetilde{S}^{-1} \widetilde{R}_{D} \widehat{S}\left[\begin{array}{c}
\lambda_{\Gamma} \\
p_{0}
\end{array}\right]=\widetilde{R}_{D}^{T} \widetilde{S}^{-1} \widetilde{R}_{D}\left[\begin{array}{c}
g_{\Gamma} \\
0
\end{array}\right] .
$$

The matrix $\widehat{S}$ defined in (3.2) is symmetric indefinite on the space $\left(\widehat{\Lambda}_{\Gamma}, W_{0}\right)$, but it is positive definite on the subspace $\left(\widehat{\Lambda}_{\Gamma} \cap \operatorname{ker}\left(\widehat{B}_{0 \Gamma}\right), W_{0}\right)$. With the carefully chosen primal velocity space $\widehat{\Lambda}_{\Pi}$, we can construct a BDDC preconditioner to make sure that the preconditioned BDDC operator in (4.2) is symmetric positive definite in a subspace and that the conjugate gradient iterates remain in this subspace when solving (4.2). This subspace is called the benign subspace.

Definition 4.1 (Benign Subspaces). We will call

$$
\widehat{\boldsymbol{\Lambda}}_{\Gamma, B}=\left\{\boldsymbol{\lambda}_{\Gamma} \in \widehat{\boldsymbol{\Lambda}}_{\Gamma} \mid \widehat{B}_{0 \Gamma} \boldsymbol{\lambda}_{\Gamma}=0\right\}, \quad \widetilde{\boldsymbol{\Lambda}}_{\Gamma, B}=\left\{\boldsymbol{\lambda}_{\Gamma} \in \widetilde{\boldsymbol{\Lambda}}_{\Gamma} \mid \widetilde{B}_{0 \Gamma} \boldsymbol{\lambda}_{\Gamma}=0\right\}
$$

the benign subspaces of $\widehat{\Lambda}_{\Gamma}$ and $\widetilde{\Lambda}_{\Gamma}$, respectively.

It is easy to see that the operators $\widehat{S}$ and $\widetilde{S}$, defined in (3.2) and (4.2), are symmetric positive definite on $\left(\widehat{\Lambda}_{\Gamma, B}, W_{0}\right)$ and $\left(\widetilde{\Lambda}_{\Gamma, B}, W_{0}\right)$, respectively.

As in $[20,28]$, in order to keep the iterates in the benign subspace, we require that the functions in the dual velocity space satisfies the no-net-flux condition, i.e.,

$$
\int_{\partial \Omega_{i}} \boldsymbol{\lambda}_{\Delta}^{(i)} \cdot \boldsymbol{n}=0, \quad \forall \boldsymbol{\lambda}_{\Delta}^{(i)} \in \boldsymbol{\Lambda}_{\Delta}^{(i)}
$$

In order to make the dual velocity space satisfy the no-net-flux condition, we choose the primal variables, which are spanned by subdomain interface edge/face basis functions with constant values at the nodes of the edge/face for two/three dimensions. We change the variables so that the degrees of freedom of each primal constraint is explicit; see [15, 21]. The dual space $\Lambda_{\triangle}$ is spanned by the remaining interface degrees of freedom with a zero average over each edge/face.

The following lemma is crucial to prove the positive definiteness of the preconditioned BDDC operator; see [20, Lemma 6.2] and [28, Lemma 4.1].

Lemma 4.2. Let $\lambda_{\Gamma} \in \widetilde{\Lambda}_{\Gamma, B}$. Then, $\widetilde{R}_{D, \Gamma}^{T} \lambda_{\Gamma} \in \widehat{\Lambda}_{\Gamma, B}$.

5. Some auxiliary results. In this section, we provide some auxiliary results for the condition number estimation for the BDDC preconditioned operator in (4.2) with different choices of the local stabilization parameter $\tau_{\kappa}$. By [37, Theorem 6.1], we know that the condition number of the elliptic problem depends on $\gamma_{h, \tau}=\max _{\kappa \in \mathcal{T}_{h}}\left\{1+\tau_{\kappa} h_{\kappa}\right\}$. Therefore, we will only estimate the condition numbers for any $\tau_{\kappa} \leq \frac{1}{h_{\kappa}}$, which covers the three choices $\tau_{k}=\frac{1}{h_{k}}, \tau_{k}=1$, and $\tau_{k}=h_{k}$ used in [24] for the numerical experiments. We will use $c$ and $C$ to indicate constants which are independent of $h, H$, and $\tau_{\kappa}$.

The subdomain matrix for $A_{a}$ in (2.6) is denoted as $A_{a}^{(i)}$ and defined as follows:

$$
A_{a}^{(i)}=\left[\begin{array}{cccc}
A_{\mathrm{LL}}^{(i)} & A_{\mathrm{uL}}^{(i)^{T}} & A_{I \mathbf{L}}^{(i)} & A_{\Gamma \mathbf{L}}^{(i) T} \\
A_{\mathrm{uL}}^{(i)} & A_{\mathbf{u u}}^{(i)} & A_{I \mathbf{u}}^{(i)} & A_{\Gamma \mathbf{u}}^{(i)} \\
A_{I \mathbf{L}}^{(i)} & A_{I \mathbf{u}}^{(i)} & A_{I I}^{(i)} & A_{\Gamma I}^{(i) T} \\
A_{\Gamma \mathbf{L}}^{(i)} & A_{\Gamma \mathbf{u}}^{(i)} & A_{\Gamma I}^{(i)} & A_{\Gamma \Gamma}^{(i)}
\end{array}\right] .
$$


We have the following result:

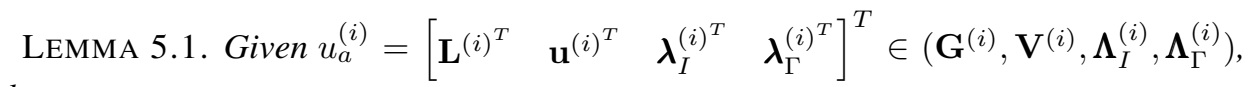
we have

$$
\left|u_{a}^{(i)}\right|_{A_{a}^{(i)}}^{2}=F\left(u_{a}\right)+\sum_{\kappa \in \mathcal{T}_{h}\left(\Omega_{i}\right)} \tau_{\kappa}\left\langle\mathbf{u}^{(i)}-\boldsymbol{\lambda}^{(i)}, \mathbf{u}^{(i)}-\boldsymbol{\lambda}^{(i)}\right\rangle_{\partial \kappa},
$$

where $\boldsymbol{\lambda}^{(i)}=\left[\begin{array}{ll}\lambda_{I}^{(i)^{T}} & \lambda_{\Gamma}^{(i)^{T}}\end{array}\right]^{T}$ and $F\left(u_{a}\right)$ is a function independent of $\tau_{\kappa}$. Moreover, $B_{a}$ defined in (2.6) is independent of $\tau_{\kappa}$.

Proof. Let $\lambda^{(i)}=\left[\begin{array}{ll}\lambda_{I}^{(i)^{T}} & \lambda_{\Gamma}^{(i)^{T}}\end{array}\right]^{T}$. By the definition in (2.4), we have

$$
\begin{aligned}
\left|u_{a}^{(i)}\right|_{A_{a}^{(i)}}^{2}= & {\left[\begin{array}{c}
\mathbf{L}^{(i)} \\
\mathbf{u}^{(i)} \\
\lambda_{I}^{(i)} \\
\lambda_{\Gamma}^{(i)}
\end{array}\right]^{T}\left[\begin{array}{cccc}
A_{\mathbf{L L}}^{(i)} & A_{\mathbf{u L}}^{(i)} & A_{I \mathbf{L}}^{(i)} & A_{\Gamma \mathbf{L}}^{(i) T} \\
A_{\mathrm{uL}}^{(i)} & A_{\mathbf{u u}}^{(i)} & A_{I \mathbf{u}}^{(i)} & A_{\Gamma \mathbf{u}}^{(i)} \\
A_{I \mathbf{L}}^{(i)} & A_{I \mathbf{u}}^{(i)} & A_{I I}^{(i)} & A_{\Gamma I}^{(i) T} \\
A_{\Gamma \mathbf{L}}^{(i)} & A_{\Gamma \mathbf{u}}^{(i)} & A_{\Gamma I}^{(i)} & A_{\Gamma \Gamma}^{(i)}
\end{array}\right]\left[\begin{array}{c}
\mathbf{L}^{(i)} \\
\mathbf{u}^{(i)} \\
\boldsymbol{\lambda}_{I}^{(i)} \\
\boldsymbol{\lambda}_{\Gamma}^{(i)}
\end{array}\right] } \\
= & \sum_{\kappa \in \mathcal{T}_{h}\left(\Omega_{i}\right)}\left(-\left(\mathbf{L}^{(i)}, \mathbf{L}^{(i)}\right)_{\kappa}-\left(\mathbf{u}^{(i)}, \nabla \cdot \mathbf{L}^{(i)}\right)_{\kappa}+\left\langle\boldsymbol{\lambda}^{(i)}, \mathbf{L}^{(i)} \mathbf{n}\right\rangle_{\partial \kappa}\right. \\
& -\left(\nabla \cdot \mathbf{L}^{(i)}, \mathbf{u}^{(i)}\right)_{\kappa}+\left\langle\tau_{\kappa} \mathbf{u}^{(i)}, \mathbf{u}^{(i)}\right\rangle_{\partial \kappa}-\left\langle\tau_{\kappa} \mathbf{u}^{(i)}, \boldsymbol{\lambda}^{(i)}\right\rangle_{\partial \kappa} \\
& \left.+\left\langle\boldsymbol{\lambda}^{(i)}, \mathbf{L}^{(i)} \mathbf{n}\right\rangle_{\partial \kappa}-\left\langle\tau_{\kappa} \mathbf{u}^{(i)}, \boldsymbol{\lambda}^{(i)}\right\rangle_{\partial \kappa}+\left\langle\tau_{\kappa} \boldsymbol{\lambda}^{(i)}, \boldsymbol{\lambda}^{(i)}\right\rangle_{\partial \kappa}\right) \\
= & \sum_{\kappa \in \mathcal{T}_{h}\left(\Omega_{i}\right)}\left(-\left(\mathbf{L}^{(i)}, \mathbf{L}^{(i)}\right)_{\kappa}-2\left(\mathbf{u}^{(i)}, \nabla \cdot \mathbf{L}^{(i)}\right)_{\kappa}+2\left\langle\boldsymbol{\lambda}^{(i)}, \mathbf{L}^{(i)} \mathbf{n}\right\rangle_{\partial \kappa}\right) \\
& +\sum_{\kappa \in \mathcal{T}_{h}\left(\Omega_{i}\right)} \tau_{\kappa}\left\langle\mathbf{u}^{(i)}-\boldsymbol{\lambda}^{(i)}, \mathbf{u}^{(i)}-\boldsymbol{\lambda}^{(i)}\right\rangle_{\partial \kappa} \\
= & F\left(u_{a}\right)+\sum_{\kappa \in \mathcal{T}_{h}\left(\Omega_{i}\right)} \tau_{\kappa}\left\langle\mathbf{u}^{(i)}-\boldsymbol{\lambda}^{(i)}, \mathbf{u}^{(i)}-\boldsymbol{\lambda}^{(i)}\right\rangle_{\partial \kappa},
\end{aligned}
$$

where $F\left(u_{a}\right)=\sum_{\kappa \in \mathcal{T}_{h}\left(\Omega_{i}\right)}\left(-\left(\mathbf{L}^{(i)}, \mathbf{L}^{(i)}\right)_{\kappa}-2\left(\mathbf{u}^{(i)}, \nabla \cdot \mathbf{L}^{(i)}\right)_{\kappa}+2\left\langle\boldsymbol{\lambda}^{(i)}, \mathbf{L}^{(i)} \mathbf{n}\right\rangle_{\partial \kappa}\right)$, which is independent of $\tau_{\kappa}$.

By the definition of $B_{a}$ in (2.6), this matrix is independent of $\tau_{\kappa}$ since $B_{p \mathbf{u}}^{T}$ and $B_{p \lambda}^{T}$ are independent of $\tau_{\kappa}$; see (2.4).

REMARK 5.2. This lemma shows that for different choices of $\tau_{\kappa}$, the divergence-free spaces of the Stokes equation in (2.5) are the same. The norms of $A_{a}^{(i)}$, for different choices of $\tau_{\kappa}$, differ only by a factor $\left\langle\mathbf{u}^{(i)}-\boldsymbol{\lambda}^{(i)}, \mathbf{u}^{(i)}-\boldsymbol{\lambda}^{(i)}\right\rangle_{\partial \kappa}$, which is a nonnegative number.

We introduce several useful norms and semi-norms, which are defined in [20, 28]. By the inertia of the Schur complement, the subdomain Schur complements $S_{\Gamma}^{(i)}$ in (3.1) are symmetric and positive semi-definite. They are singular for any subdomains which do not intersect $\partial \Omega$. Due to the Dirichlet boundary condition on $\partial \Omega$ and sufficiently many primal continuity constraints for the no-net-flux condition, the operators $\widehat{S}_{\Gamma}$ in (3.2) and $\widetilde{S}_{\Gamma}$ in (4.1) are symmetric positive definite. The interface operators $\widehat{S}$ in (3.2) and $\widetilde{S}$ in (4.1) are indefinite, but they are positive definite when they are restricted to the respective benign subspaces of 
$\left(\widehat{\Lambda}_{\Gamma}, W_{0}\right)$ and $\left(\widetilde{\Lambda}_{\Gamma}, W_{0}\right)$. We can define

$$
\begin{array}{ll}
|\mathbf{w}|_{\widehat{S}}^{2}=\mathbf{w}^{T} \widehat{S} \mathbf{w}=\left\|\lambda_{\Gamma}\right\|_{\widehat{S}_{\Gamma}}^{2}, \quad \forall \mathbf{w}=\left(\boldsymbol{\lambda}_{\Gamma}, p_{0}\right) \in\left(\widehat{\Lambda}_{\Gamma, B}, W_{0}\right), \\
|\mathbf{w}|_{\widetilde{S}}^{2}=\mathbf{w}^{T} \widetilde{S} \mathbf{w}=\left\|\lambda_{\Gamma}\right\|_{\widetilde{S}_{\Gamma}}^{2}, \quad \forall \mathbf{w}=\left(\boldsymbol{\lambda}_{\Gamma}, p_{0}\right) \in\left(\widetilde{\Lambda}_{\Gamma, B}, W_{0}\right) .
\end{array}
$$

Among all $\tau_{\kappa} \leq \frac{1}{h_{\kappa}}$, the choice $\tau_{\kappa}=\frac{1}{h_{\kappa}}$ has some nice properties which are not shared by other choices of $\tau_{\kappa}$. Therefore, we use the subscript ${ }_{*}$ to indicate those matrices with this value of $\tau_{\kappa}=\frac{1}{h_{\kappa}}$, such as, for example, $\widehat{S}_{\Gamma, *}, \widetilde{S}_{\Gamma, *}$, and $A_{a, *}^{(i)}$.

The following results are proved in [39].

LEMMA 5.3 (Lemma A.2 in [39]). If $\tau_{k}=\frac{1}{h_{k}}$, then

$$
\sum_{\kappa \in \mathcal{T}_{h}\left(\Omega_{i}\right)}\left\|\nabla \mathbf{u}^{(i)}\right\|_{L^{2}(\kappa)}^{2} \leq C\left|\left[\begin{array}{c}
\mathbf{L}^{(i)} \\
\mathbf{u}^{(i)} \\
\lambda_{I}^{(i)} \\
\lambda_{\Gamma}^{(i)}
\end{array}\right]\right|_{A_{a, *}^{(i)}}^{2}
$$

for all $\left[\begin{array}{llll}\mathbf{L}^{(i)^{T}} & \mathbf{u}^{(i)^{T}} & \lambda_{I}^{(i)^{T}} & \lambda_{\Gamma}^{(i)^{T}}\end{array}\right]^{T} \in\left(\mathbf{G}^{(i)}, \mathbf{V}^{(i)}, \boldsymbol{\Lambda}_{I}^{(i)}, \boldsymbol{\Lambda}_{\Gamma}^{(i)}\right)$ satisfying

$$
A_{\mathbf{L} \mathbf{L}, *}^{(i)} \mathbf{L}^{(i)}+A_{u \mathbf{L}, *}^{(i)^{T}} \mathbf{u}^{(i)}+A_{I \mathbf{L}, *}^{(i)^{T}} \lambda_{I}^{(i)}+A_{\Gamma \mathbf{L}, *}^{(i)^{T}} \lambda_{\Gamma}^{(i)}=0 .
$$

LEMMA 5.4 (Lemma 4.3 in [39]). If $\tau_{\kappa}=\frac{1}{h_{\kappa}}$, then there exists a positive constant $\beta$ independent of $h$ and $H$ such that

$$
\sup _{u_{a} \in(\mathbf{G}, \mathbf{V}, \boldsymbol{\Lambda})} \frac{u_{a}^{T} B_{a, *}^{T} p}{\left(u_{a}^{T} A_{a, *} u_{a}\right)^{1 / 2}} \geq \beta\|p\|_{L^{2}(\Omega)},
$$

for all $p \in W$. Here $A_{a, *}, B_{a, *}$ are defined in (2.6) with $\tau_{\kappa}=\frac{1}{h_{\kappa}}$. The lemma also holds when $\Omega$ is replaced by the subdomain $\Omega_{i}$.

We also define $S_{\Gamma, E}^{(i)}$, the subdomain Schur complement for the corresponding elliptic problem with $\tau_{\kappa}=\frac{1}{h_{\kappa}}$ as follows: given $\boldsymbol{\lambda}_{\Gamma}^{(i)} \in \Lambda_{\Gamma}^{(i)}$, determine $S_{\Gamma, E}^{(i)} \boldsymbol{\lambda}_{\Gamma}^{(i)}$ such that

$$
A_{a, *}^{(i)}\left[\begin{array}{c}
\mathbf{L}^{(i)} \\
\mathbf{u}^{(i)} \\
\boldsymbol{\lambda}_{I}^{(i)} \\
\boldsymbol{\lambda}_{\Gamma}^{(i)}
\end{array}\right]=\left[\begin{array}{c}
\mathbf{0} \\
\mathbf{0} \\
\mathbf{0} \\
S_{\Gamma, E}^{(i)} \boldsymbol{\lambda}_{\Gamma}^{(i)}
\end{array}\right] .
$$

Let $\left|\lambda_{\Gamma}^{(i)}\right|_{S_{\Gamma}^{(i)}}^{2}=\lambda_{\Gamma}^{(i) T} S_{\Gamma}^{(i)} \lambda_{\Gamma}^{(i)}$ and $\left|\lambda_{\Gamma}^{(i)}\right|_{S_{\Gamma, E}^{(i)}}^{2}=\lambda_{\Gamma}^{(i) T} S_{\Gamma, E}^{(i)} \lambda_{\Gamma}^{(i)}$. Similar to the proof of [38, Lemma 12], we can use Lemma 5.3 and the inf-sup stability for each subdomain of Lemma 5.4 to prove the following result. Similar results for the standard finite element discretization can be found in [3].

LEMMA 5.5. If $\tau_{k}=\frac{1}{h_{k}}$, then

$$
c \frac{\beta^{2}}{(1+\beta)^{2}}\left|\lambda_{\Gamma}^{(i)}\right|_{S_{\Gamma, *}^{(i)}}^{2} \leq\left|\lambda_{\Gamma}^{(i)}\right|_{S_{\Gamma, E}^{(i)}}^{2} \leq\left|\lambda_{\Gamma}^{(i)}\right|_{S_{\Gamma, *}^{(i)}}^{2}, \quad \forall \lambda_{\Gamma}^{(i)} \in \Lambda_{\Gamma}^{(i)},
$$

where $\beta$ is the inf-sup stability constant in Lemma 5.4. 
In order to prove the condition number bounds for the BDDC preconditioner, we define an averaging operator $E_{D}$, which maps $\left(\widetilde{\Lambda}_{\Gamma}, W_{0}\right)$, with generally discontinuous interface velocities, to the same space with continuous interface velocities: for any $w=\left(\boldsymbol{\lambda}_{\Gamma}, p_{0}\right) \in$ $\left(\widetilde{\Lambda}_{\Gamma}, W_{0}\right)$,

$$
E_{D}\left[\begin{array}{l}
\lambda_{\Gamma} \\
p_{0}
\end{array}\right]=\left[\begin{array}{ll}
\widetilde{R}_{\Gamma} & \\
& I
\end{array}\right]\left[\begin{array}{ll}
\widetilde{R}_{D, \Gamma}^{T} & \\
& I
\end{array}\right]\left[\begin{array}{c}
\lambda_{\Gamma} \\
p_{0}
\end{array}\right]=\left[\begin{array}{c}
E_{D, \Gamma} \boldsymbol{\lambda}_{\Gamma} \\
p_{0}
\end{array}\right] \in\left(\widetilde{\boldsymbol{\Lambda}}_{\Gamma}, W_{0}\right),
$$

where $E_{D, \Gamma}=\widetilde{R}_{\Gamma} \widetilde{R}_{D, \Gamma}^{T}$ averages the interface velocity with a properly defined weight.

The following lemma is the result for $E_{D, \Gamma}$; for the elliptic problem with $\tau_{\kappa}=\frac{1}{h_{\kappa}}$, see [37, Lemma 5].

LEMMA 5.6. There exists a positive constant $C$, which is independent of $H$ and $h$, such that

$$
\left|E_{D, \Gamma} \boldsymbol{\lambda}_{\Gamma}\right|_{\widetilde{S}_{\Gamma, E}}^{2} \leq C\left(1+\log \frac{H}{h}\right)^{2}\left|\boldsymbol{\lambda}_{\Gamma}\right|_{\widetilde{S}_{\Gamma, E}}^{2}, \quad \forall \boldsymbol{\lambda}_{\Gamma} \in \widetilde{\boldsymbol{\Lambda}}_{\Gamma}
$$

We define the harmonic and Stokes extensions from $\Lambda_{\Gamma}^{(i)}$ to $\left(\mathbf{G}^{(i)}, \mathbf{V}^{(i)}, \Lambda^{(i)}\right)$ as follows: for any $\lambda_{\Gamma}^{(i)} \in \Lambda_{\Gamma}^{(i)}$,

$$
\left|\mathcal{H} \lambda_{\Gamma}^{(i)}\right|_{A_{a}^{(i)}} \inf _{\mathbf{E}^{(i)}=\left(\mathbf{L}^{(i)}, \mathbf{v}^{(i)}, \boldsymbol{\lambda}^{(i)}\right) \in\left(\mathbf{G}^{(i)}, \mathbf{V}^{(i)}, \mathbf{\Lambda}^{(i)}\right),\left.\mathbf{E}^{(i)}\right|_{\Gamma_{i}}=\boldsymbol{\lambda}_{\Gamma}^{(i)}}\left|\mathbf{E}^{(i)}\right|_{A_{a}^{(i)}},
$$

and

$$
\left|\mathcal{S} \boldsymbol{\lambda}_{\Gamma}^{(i)}\right|_{A_{a}^{(i)}}=\inf _{\mathbf{E}^{(i)}=\left(\mathbf{L}^{(i)}, \mathbf{V}^{(i)}, \boldsymbol{\lambda}^{(i)}\right) \in\left(\mathbf{G}^{(i)}, \mathbf{V}^{(i)}, \boldsymbol{\Lambda}^{(i)}\right), \mathbf{E}^{(i)} \mid \Gamma_{i}=\boldsymbol{\lambda}_{\Gamma}^{(i)}, B_{a, c}^{(i)} \mathbf{E}^{(i)}=0}\left|\mathbf{E}^{(i)}\right|_{A_{a}^{(i)},},
$$

where $A_{a}^{(i)}$, defined in (5.1), is the subdomain local matrix of $A_{a}$ in (2.6). $B_{a, c}^{(i)}$ is the subdomain local matrix of $B_{a}$ in (2.6), excluding the pressure degree of freedom corresponding to the subdomain average pressure. Given $\lambda_{\Gamma}^{(i)}$, the harmonic extension $\mathcal{H} \lambda_{\Gamma}^{(i)}$ can be obtained by solving the equations corresponding to the first three rows of (5.2). The Stokes extension $\mathcal{S} \lambda_{\Gamma}^{(i)}$ can be obtained by solving the subdomain version of (2.5) with given $\lambda_{\Gamma}^{(i)}$.

By the definition of these two extensions and the semi-norms $|\cdot|_{S_{\Gamma, E}}$ and $|\cdot|_{S_{\Gamma}}$, we have

$$
\left|\mathcal{H}_{*} \lambda_{\Gamma}^{(i)}\right|_{A_{a, *}^{(i)}}=\left|\lambda_{\Gamma}^{(i)}\right|_{S_{\Gamma, E}^{(i)}}^{2}, \quad\left|\mathcal{S}_{*} \lambda_{\Gamma}^{(i)}\right|_{A_{a, *}^{(i)}}=\left|\lambda_{\Gamma}^{(i)}\right|_{S_{\Gamma, *}^{(i)}}^{2}, \quad\left|\mathcal{S} \lambda_{\Gamma}^{(i)}\right|_{A_{a}^{(i)}}=\left|\lambda_{\Gamma}^{(i)}\right|_{S_{\Gamma}^{(i)}}^{2} .
$$

Recall that $\mathcal{S}_{*} \lambda_{\Gamma}^{(i)}$ is the Stokes extension with $\tau_{\kappa}=\frac{1}{h_{\kappa}}$. By [37, Lemma 5.1], we have the following result.

LEMMA 5.7. For $\tau_{\kappa} \leq \frac{1}{h_{k}}, \forall \boldsymbol{\lambda}^{(i)} \in \boldsymbol{\Lambda}^{(i)}$, we have

$$
\left|\boldsymbol{\lambda}^{(i)}\right|_{A_{a, *}^{(i)}} \leq C\left|\boldsymbol{\lambda}^{(i)}\right|_{A_{a}^{(i)}} .
$$

Proof. For any $\lambda \in \Lambda^{(i)}$, as in [5, 11,37], let $|\partial \kappa|$ denote the measure (the length for 2D and area for 3D) of the boundary of $\kappa$. Define $m_{\kappa}=\frac{1}{|\partial \kappa|} \int_{\partial \kappa} \lambda d s$ and

$$
\|\boldsymbol{\lambda}\|_{\Omega_{i}}=\left(\sum_{\kappa \in \mathcal{T}_{h}, \kappa \subseteq \overline{\Omega_{i}}} \frac{1}{h_{\kappa}}\left\|\boldsymbol{\lambda}-m_{\kappa}(\boldsymbol{\lambda})\right\|_{L^{2}(\partial \kappa)}^{2}\right)^{1 / 2} .
$$


By [37, Lemma 5.1], we have

$$
\left|\lambda^{(i)}\right|_{A_{a, *}^{(i)}} \leq\left. C||\left|\lambda^{(i)}\right|\right|_{\Omega_{i}} ^{2} \leq C\left|\lambda^{(i)}\right|_{A_{a}^{(i)}}^{2} .
$$

Now, we are ready to prove the bound for the averaging operator $E_{D}$ for the Stokes problem for all $\tau_{\kappa} \leq \frac{1}{h_{\kappa}}$.

LEMMA 5.8. If $\tau_{\kappa} \leq \frac{1}{h_{\kappa}}$, then there exists a positive constant $C$, which is independent of $H$ and $h$, such that

$$
\left|E_{D} w\right|_{\widetilde{S}}^{2} \leq C \frac{(1+\beta)^{2}}{\beta^{2}}\left(1+\log \frac{H}{h}\right)^{2}|w|_{\widetilde{S}}^{2}, \quad \forall w=\left(\boldsymbol{\lambda}_{\Gamma}, p_{0}\right) \in\left(\widetilde{\Lambda}_{\Gamma, B}, W_{0}\right),
$$

where $\beta$ is the inf-sup stability constant in Lemma 5.4.

Proof. For any vector $w=\left(\boldsymbol{\lambda}_{\Gamma}, p_{0}\right) \in\left(\widetilde{\boldsymbol{\Lambda}}_{\Gamma, B}, W_{0}\right)$, by Lemma 4.2 it follows that $\widetilde{R}_{D, \Gamma}^{T} \boldsymbol{\lambda}_{\Gamma} \in \widehat{\Lambda}_{\Gamma, B}$. Thus, $E_{D, \Gamma} \boldsymbol{\lambda}_{\Gamma}=\widetilde{R}_{\Gamma} \widetilde{R}_{D, \Gamma}^{T} \lambda_{\Gamma} \in \widetilde{\Lambda}_{\Gamma, B}$.

Let $\mu_{\Gamma}^{(i)}=\bar{R}_{\Gamma}^{(i)} E_{D, \Gamma} \lambda_{\Gamma}$. By the definition of the $\widetilde{S}$-seminorm, we have that

$$
\left|E_{D} w\right|_{\widetilde{S}}^{2} \leq \sum_{i=1}^{N}\left|\mathcal{S}_{*} \boldsymbol{\mu}_{\Gamma}^{(i)}\right|_{A_{a}^{(i)}}^{2}
$$

since

$$
\begin{aligned}
\left|E_{D} w\right|_{\widetilde{S}}^{2} & =\left|E_{D, \Gamma} \lambda_{\Gamma}\right|_{\widetilde{S}_{\Gamma}}^{2}=\sum_{i=1}^{N}\left|\bar{R}_{\Gamma}^{(i)} E_{D, \Gamma} \boldsymbol{\lambda}_{\Gamma}\right|_{S_{\Gamma}^{(i)}}^{2} \\
& =\sum_{i=1}^{N}\left|\boldsymbol{\mu}_{\Gamma}^{(i)}\right|_{S_{\Gamma}^{(i)}}^{2}=\sum_{i=1}^{N}\left|\mathcal{S} \boldsymbol{\mu}_{\Gamma}^{(i)}\right|_{A_{a}^{(i)}}^{2} \leq \sum_{i=1}^{N}\left|\mathcal{S}_{*} \mu_{\Gamma}^{(i)}\right|_{A_{a}^{(i)}}^{2},
\end{aligned}
$$

where we used the minimization property of $\mathcal{S} \boldsymbol{\mu}_{\Gamma}^{(i)}$, the fact that $B_{a}$ is independent of $\tau_{k}$ in Lemma 5.1, which gives that the divergence-free spaces for different $\tau_{\kappa}$ are the same, and the definition of $\mathcal{S}_{*} \boldsymbol{\mu}_{\Gamma}^{(i)}$.

$$
\begin{aligned}
\text { Let } \mathcal{S}_{*} \boldsymbol{\mu}_{\Gamma}^{(i)}= & {\left[\begin{array}{c}
\mathbf{L}^{(i)} \\
\mathbf{u}^{(i)} \\
\boldsymbol{\mu}_{I}^{(i)} \\
\boldsymbol{\mu}_{\Gamma}^{(i)}
\end{array}\right] \text {. By (5.4) and Lemma 5.1, we have } } \\
\left|E_{D} w\right|_{\widetilde{S}}^{2} & \leq \sum_{i=1}^{N}\left|\mathcal{S}_{*} \boldsymbol{\mu}_{\Gamma}^{(i)}\right|_{A_{a}^{(i)}}^{2} \\
& =\sum_{i=1}^{N}\left(F\left(\mathcal{S}_{*} \boldsymbol{\mu}_{\Gamma}^{(i)}\right)+\sum_{\kappa \in \mathcal{T}_{h}\left(\Omega_{i}\right)} \tau_{\kappa}\left\langle\mathbf{u}^{(i)}-\boldsymbol{\mu}^{(i)}, \mathbf{u}^{(i)}-\boldsymbol{\mu}^{(i)}\right\rangle_{\partial \kappa}\right) \\
& \leq \sum_{i=1}^{N}\left(F\left(\mathcal{S}_{*} \boldsymbol{\mu}_{\Gamma}^{(i)}\right)+\sum_{\kappa \in \mathcal{T}_{h}\left(\Omega_{i}\right)} \frac{1}{h_{\kappa}}\left\langle\mathbf{u}^{(i)}-\boldsymbol{\mu}^{(i)}, \mathbf{u}^{(i)}-\boldsymbol{\mu}^{(i)}\right\rangle_{\partial \kappa}\right) \\
& =\sum_{i=1}^{N}\left|\mathcal{S}_{*} \boldsymbol{\mu}_{\Gamma}^{(i)}\right|_{A_{a, *}^{(i)}}^{2}=\sum_{i=1}^{N}\left|\boldsymbol{\mu}_{\Gamma}^{(i)}\right|_{S_{\Gamma, *}^{(i)}}^{2} \leq \frac{(1+\beta)^{2}}{c \beta^{2}} \sum_{i=1}^{N}\left|\boldsymbol{\mu}_{\Gamma}^{(i)}\right|_{S_{\Gamma, E}^{(i)}}^{2},
\end{aligned}
$$


where we used Lemma 5.1 for the second and fourth equalities, (5.3) for the fifth equality, and Lemma 5.5 for the sixth inequality.

By Lemma 5.6, the estimate of the average operator for the elliptic problem, we have

$$
\begin{aligned}
\left|E_{D} w\right|_{\widetilde{S}}^{2} & \leq \frac{(1+\beta)^{2}}{c \beta^{2}} \sum_{i=1}^{N}\left|\boldsymbol{\mu}_{\Gamma}^{(i)}\right|_{S_{\Gamma, E}^{(i)}}^{2} \leq C \frac{(1+\beta)^{2}}{\beta^{2}}\left(1+\log \frac{H}{h}\right)^{2}\left|\lambda_{\Gamma}\right|_{\widetilde{S}_{\Gamma, E}}^{2} \\
& =C \frac{(1+\beta)^{2}}{\beta^{2}}\left(1+\log \frac{H}{h}\right)^{2} \sum_{i=1}^{N}\left|\lambda_{\Gamma}^{(i)}\right|_{S_{\Gamma, E}^{(i)}}^{2} \\
& =C \frac{(1+\beta)^{2}}{\beta^{2}}\left(1+\log \frac{H}{h}\right)^{2} \sum_{i=1}^{N}\left|\mathcal{H}_{*} \lambda_{\Gamma}^{(i)}\right|_{A_{a, *}^{(i)}}^{2} \\
& \leq C \frac{(1+\beta)^{2}}{\beta^{2}}\left(1+\log \frac{H}{h}\right)^{2} \sum_{i=1}^{N}\left|\mathcal{H} \lambda_{\Gamma}^{(i)}\right|_{A_{a, *}^{(i)}}^{2} \\
& \leq C \frac{(1+\beta)^{2}}{\beta^{2}}\left(1+\log \frac{H}{h}\right)^{2} \sum_{i=1}^{N}\left|\mathcal{H} \lambda_{\Gamma}^{(i)}\right|_{A_{a}^{(i)}}^{2} \\
& \leq C \frac{(1+\beta)^{2}}{\beta^{2}}\left(1+\log \frac{H}{h}\right)^{2} \sum_{i=1}^{N}\left|\mathcal{S} \lambda_{\Gamma}^{(i)}\right|_{A_{a}^{(i)}}^{2} \\
& \leq C \frac{(1+\beta)^{2}}{\beta^{2}}\left(1+\log \frac{H}{h}\right)^{2}\left|\lambda_{\Gamma}\right|_{\widetilde{S}_{\Gamma}}^{2}
\end{aligned}
$$

where we used (5.3) for (5.5) and the minimization property of the harmonic extension $\mathcal{H}_{*}$ for (5.6). Lemma 5.7 is used in (5.7). The last two inequalities are obtained by using the definitions of the harmonic and Stokes extensions and (5.3).

Finally, we have

$$
\left|E_{D} w\right|_{\widetilde{S}}^{2} \leq C \frac{(1+\beta)^{2}}{\beta^{2}}\left(1+\log \frac{H}{h}\right)^{2}\left|\lambda_{\Gamma}\right|_{\widetilde{S}_{\Gamma}}^{2} \leq C \frac{(1+\beta)^{2}}{\beta^{2}}\left(1+\log \frac{H}{h}\right)^{2}|w|_{\widetilde{S}}^{2} .
$$

6. Condition number estimate for the BDDC preconditioner. We are now ready to formulate our main result. The proof follows that of [20, Theorem 6.7], [23, Theorem 25], [32, Lemma 4.6], and [31, Lemma 4.7] by using Lemma 5.8. For the sake of completeness, we provide the proof here.

THEOREM 6.1. The preconditioned operator $M^{-1} \widehat{S}$ is symmetric, positive definite with respect to the bilinear form $\langle\cdot, \cdot\rangle_{\widehat{S}}$ on the space $\left(\widehat{\Lambda}_{\Gamma, B}, W_{0}\right)$. If $\tau_{\kappa} \leq \frac{1}{h_{\kappa}}$, then the condition number of $M^{-1} \widehat{S}$ is bounded by $C \frac{(1+\beta)^{2}}{\beta^{2}}\left(1+\log \left(\frac{H}{h}\right)\right)^{2}$, where $C$ is a constant, which is independent of $H$ and $h$, and $\beta$ is the inf-sup stability constant, defined in Lemma 5.4.

Proof. We only need to prove that, for any $\mathbf{u} \in\left(\widehat{\boldsymbol{\Lambda}}_{\Gamma, B}, W_{0}\right)$, it holds that

$$
\langle\mathbf{u}, \mathbf{u}\rangle_{\widehat{S}} \leq\left\langle\mathbf{u}, M^{-1} \widehat{S} \mathbf{u}\right\rangle_{\widehat{S}} \leq C \frac{(1+\beta)^{2}}{\beta^{2}}(1+\log (H / h))^{2}\langle\mathbf{u}, \mathbf{u}\rangle_{\widehat{S}}
$$

Lower bound: Let

$$
\mathbf{w}=\widetilde{S}^{-1} \widetilde{R}_{D} \widehat{S} \mathbf{u} \in\left(\widehat{\Lambda}_{\Gamma, B}, W_{0}\right)
$$


We then have that

$$
\begin{aligned}
\mathbf{u}^{T} \widehat{S} \mathbf{u} & =\mathbf{u}^{T} \widehat{S} \widetilde{R}_{D}^{T} \widetilde{R} \mathbf{u}=\mathbf{u}^{T} \widehat{S} \widetilde{R}_{D}^{T} \widetilde{S}^{-1} \widetilde{S} \widetilde{R} \mathbf{u}=\langle\mathbf{w}, \widetilde{R} \mathbf{u}\rangle_{\widetilde{S}} \\
& \leq\left(\mathbf{u}^{T} \widetilde{R}^{T} \widetilde{S} \widetilde{R} \mathbf{u}\right)^{1 / 2}\left(\mathbf{w}^{T} \widetilde{S} \mathbf{w}\right)^{1 / 2} \\
& =\left(\mathbf{u}^{T} \widehat{S} \mathbf{u}\right)^{1 / 2}\left(\mathbf{u}^{T} \widehat{S} \widetilde{R}_{D}^{T} \widetilde{S}^{-1} \widetilde{S} \widetilde{S}^{-1} \widetilde{R}_{D} \widehat{S} \mathbf{u}\right)^{1 / 2} \\
& =\left(\mathbf{u}^{T} \widehat{S} \mathbf{u}\right)^{1 / 2}\left(\mathbf{u}^{T} \widehat{S} M^{-1} \widehat{S} \mathbf{u}\right)^{1 / 2},
\end{aligned}
$$

where we use the property $\widetilde{R}^{T} \widetilde{R}_{D}=\widetilde{R}_{D}^{T} \widetilde{R}=I$ for the first equality and (6.1) for the third equality.

Simplifying a common factor and squaring, we obtain

$$
\langle\mathbf{u}, \mathbf{u}\rangle_{\widehat{S}} \leq\left\langle\mathbf{u}, M^{-1} \widehat{S} \mathbf{u}\right\rangle_{\widehat{S}}
$$

Upper bound: The upper bound can be estimated by using the definition of $\mathbf{w}$ in (6.1), the Cauchy-Schwarz inequality, and Lemma 5.8:

$$
\begin{aligned}
\left\langle\mathbf{u}, M^{-1} \widehat{S} \mathbf{u}\right\rangle_{\widehat{S}} & \leq\langle\mathbf{u}, \mathbf{u}\rangle_{\widehat{S}}^{1 / 2}\left\langle M^{-1} \widehat{S} \mathbf{u}, M^{-1} \widehat{S} \mathbf{u}\right\rangle_{\widehat{S}}^{1 / 2}=\langle\mathbf{u}, \mathbf{u}\rangle_{\widehat{S}}^{1 / 2}\left\langle\widetilde{R}_{D}^{T} \mathbf{w}, \widetilde{R}_{D}^{T} \mathbf{w}\right)_{\widehat{S}}^{1 / 2} \\
& =\langle\mathbf{u}, \mathbf{u}\rangle_{\widehat{S}}^{1 / 2}\left\langle\widetilde{R} \widetilde{R}_{D}^{T} \mathbf{w}, \widetilde{R} \widetilde{R}_{D}^{T} \mathbf{w}\right)_{\widetilde{S}}^{1 / 2}=\langle\mathbf{u}, \mathbf{u}\rangle_{\widehat{S}}^{1 / 2}\left|E_{D} \mathbf{w}\right|_{\widetilde{S}} \\
& \leq C\langle\mathbf{u}, \mathbf{u}\rangle_{\widehat{S}}^{1 / 2} \frac{(1+\beta)}{\beta}(1+\log (H / h))|\mathbf{w}|_{\widetilde{S}} \\
& =C \frac{(1+\beta)}{\beta}(1+\log (H / h))\langle\mathbf{u}, \mathbf{u}\rangle_{\widehat{S}}^{1 / 2}\left(\mathbf{u}^{T} \widehat{S} \widetilde{R}_{D}^{T} \widetilde{S}^{-1} \widetilde{S}_{S^{-1}} \widetilde{R}_{D} \widehat{S} \mathbf{u}\right)^{1 / 2} \\
& =C \frac{(1+\beta)}{\beta}(1+\log (H / h))\langle\mathbf{u}, \mathbf{u}\rangle_{\widehat{S}}^{1 / 2}\left\langle\mathbf{u}, M^{-1} \widehat{S} \mathbf{u}\right\rangle_{\widehat{S}}^{1 / 2}
\end{aligned}
$$

Therefore,

$$
\left\langle\mathbf{u}, M^{-1} \widehat{S} \mathbf{u}\right\rangle_{\widehat{S}} \leq C \frac{(1+\beta)^{2}}{\beta^{2}}(1+\log (H / h))^{2}\langle\mathbf{u}, \mathbf{u}\rangle_{\widehat{S}}
$$

7. Numerical experiments. In our numerical experiments, we consider a unit square domain $\Omega$, i.e., $\Omega=[0,1]^{2}$. The domain is then decomposed into $N \times N$ subdomains with side length $H=1 / N$, and each subdomain is further decomposed into elements with diameter $h$. The model problem (2.1) is discretized by the $k$ th-order HDG method. Here, zero Dirichlet boundary conditions are used, and we choose the right-hand side function $f$ such that the exact solution is

$$
\mathbf{u}=\left[\begin{array}{c}
\sin ^{3}(\pi x) \sin ^{2}(\pi y) \cos (\pi y) \\
-\sin ^{2}(\pi x) \sin ^{3}(\pi y) \cos (\pi x)
\end{array}\right] \text { and } p=x-y .
$$

The preconditioned conjugate gradient iteration is stopped when the relative $l_{2}$-norm of the residual has been reduced by a factor of $10^{6}$.

Following [24], three different choices of the stabilization constant $\tau_{\kappa}$ are tested, namely $\tau_{\kappa}=h_{\kappa}, \tau_{\kappa}=1$, and $\tau_{\kappa}=\frac{1}{h_{\kappa}}$. Two sets of experiments are carried out for each choice of $\tau_{\kappa}$ 
TABLE 7.1

Performance of solving (4.2) with the HDG discretization $\left(\tau_{\kappa}=h_{k}\right)$.

\begin{tabular}{cccccccccc} 
& & \multicolumn{2}{c}{$k=0$} & & \multicolumn{2}{c}{$k=1$} & & \multicolumn{2}{c}{$k=2$} \\
\cline { 3 - 4 }$H / h$ & \#sub & Cond. & Iter. & & Cond. & Iter. & & Cond. & Iter. \\
\hline 8 & $4 \times 4$ & 4.12 & 10 & & 4.46 & 12 & & 12.40 & 14 \\
& $8 \times 8$ & 5.03 & 13 & & 8.33 & 16 & & 11.27 & 20 \\
& $16 \times 16$ & 4.88 & 13 & & 9.86 & 20 & & 13.16 & 24 \\
& $24 \times 24$ & 5.04 & 13 & & 10.26 & 20 & & 13.67 & 24 \\
& $32 \times 32$ & 4.94 & 12 & & 10.23 & 19 & & 13.77 & 24 \\
\hline \#sub & $H / h$ & Cond. & Iter. & & Cond. & Iter. & & Cond. & Iter. \\
\hline $8 \times 8$ & 4 & 2.49 & 9 & & 5.85 & 13 & & 8.31 & 17 \\
& 8 & 5.03 & 13 & & 8.33 & 16 & & 11.27 & 20 \\
& 16 & 7.48 & 15 & & 11.28 & 20 & & 17.45 & 24 \\
& 24 & 9.12 & 17 & & 13.22 & 21 & & 20.00 & 25 \\
& 32 & 10.37 & 19 & & 17.28 & 23 & & 21.82 & 26
\end{tabular}

TABLE 7.2

Performance of solving (4.2) with the HDG discretization $\left(\tau_{\kappa}=1\right)$.

\begin{tabular}{cccccccccc} 
& & \multicolumn{2}{c}{$k=0$} & & \multicolumn{2}{c}{$k=1$} & & \multicolumn{2}{c}{$k=2$} \\
\cline { 3 - 4 }$H / h$ & \#sub & Cond. & Iter. & & Cond. & Iter. & & Cond. & Iter. \\
\hline 8 & $4 \times 4$ & 4.13 & 10 & & 4.46 & 12 & & 12.12 & 14 \\
& $8 \times 8$ & 5.03 & 13 & & 8.34 & 17 & & 11.27 & 20 \\
& $16 \times 16$ & 4.88 & 13 & & 9.87 & 20 & & 13.16 & 24 \\
& $24 \times 24$ & 5.04 & 13 & & 10.26 & 20 & & 13.67 & 24 \\
& $32 \times 32$ & 4.94 & 12 & & 10.23 & 19 & & 13.77 & 24 \\
\hline \#sub & $H / h$ & Cond. & Iter. & & Cond. & Iter. & & Cond. & Iter. \\
\hline $8 \times 8$ & 4 & 2.49 & 9 & & 5.86 & 13 & & 8.32 & 17 \\
& 8 & 5.03 & 13 & & 8.34 & 17 & & 11.27 & 20 \\
& 16 & 7.49 & 15 & & 11.28 & 20 & & 17.51 & 24 \\
& 24 & 9.12 & 17 & & 13.22 & 21 & & 19.83 & 25 \\
& 32 & 10.37 & 19 & & 14.69 & 22 & & 21.15 & 25
\end{tabular}

to obtain iteration counts and condition number estimates. In the first set of experiments, we fixed $\frac{H}{h}=8$, the subdomain local problem size, and increased the number of subdomains to test the scalability of the algorithms (that the condition number is independent of the number of subdomains). In the second set of experiments, we fixed the number of subdomains to 64 and changed $\frac{H}{h}$, the subdomain local problem size. The performance of the algorithms for the Stokes problem with these three choices of $\tau_{\kappa}$ is similar to that for the elliptic problems. The experimental results are fully consistent with our theory. In Figure 7.1, we present the least-square fits of the condition numbers to the function $C\left(1+\log \left(\frac{H}{h}\right)\right)^{2}$ with $\tau_{\kappa}=h_{\kappa}$ and 64 subdomains. The results with other choices of $\tau_{\kappa}$ are similar. We also use the condition numbers for $\frac{H}{h}=8$ to conjecture that the condition numbers depend on the polynomial order $k$ as $C_{1} \log \left(k+C_{0}\right)$. In Figure 7.2, we display the least-square fits of the condition numbers to $C_{1} \log \left(k+C_{0}\right)$ with $\tau_{\kappa}=h_{\kappa}$. 

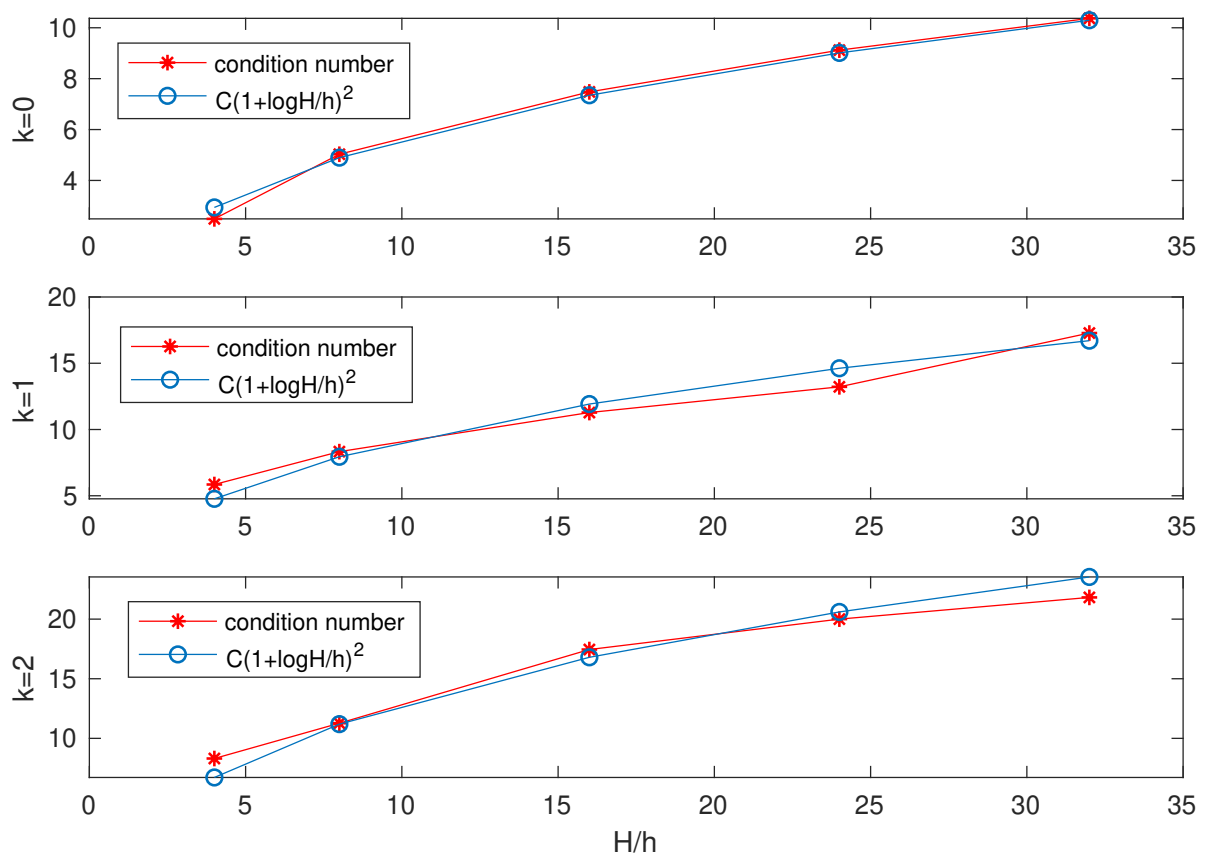

FIG. 7.1. Least square fits of the condition numbers in Table 7.1 with respect to $H / h$ to the function $C(1+\log (H / h))^{2}$ for $\tau_{\kappa}=h_{\kappa}$.
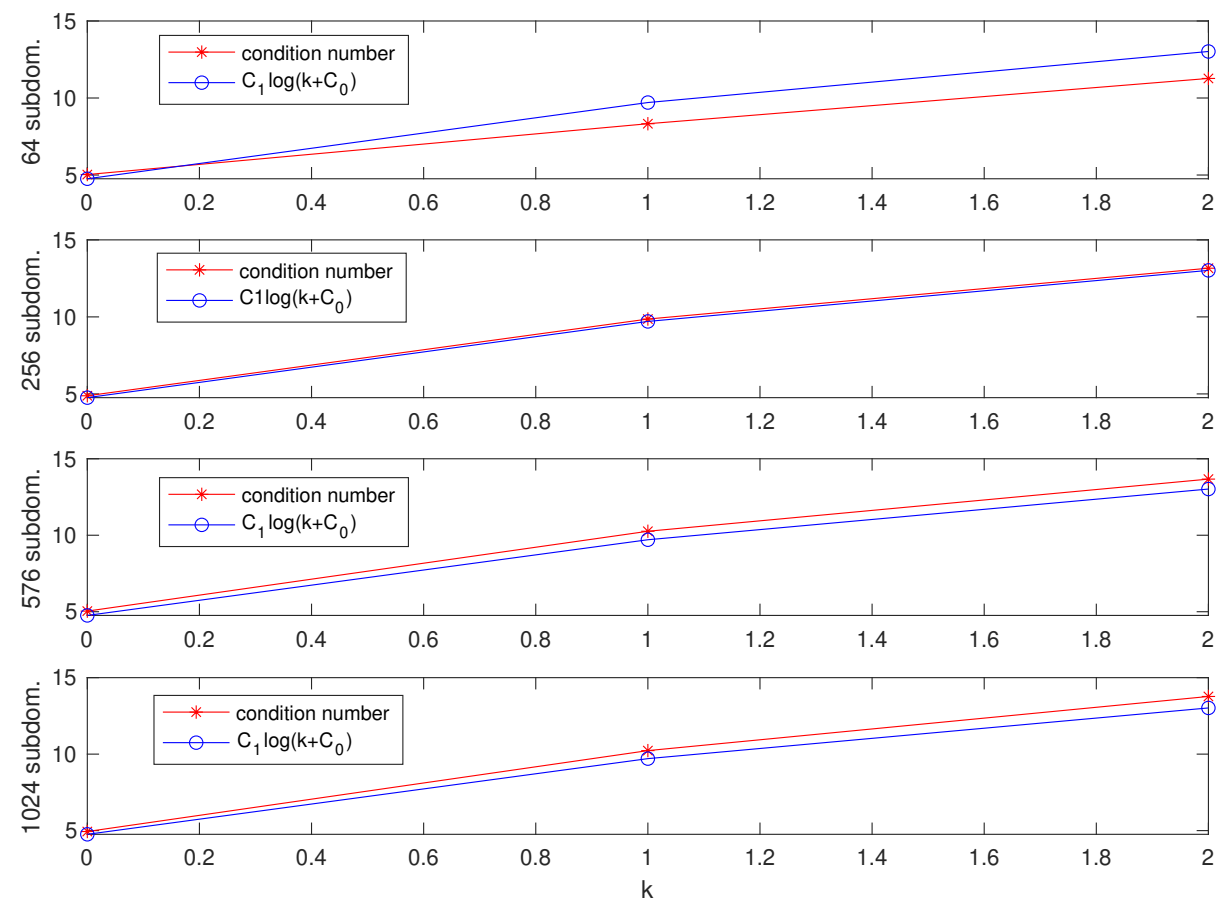

FIG. 7.2. Least square fits of the condition numbers in Table 7.1 with respect to $k$ to the function $C_{1} \log \left(k+C_{0}\right)$ for different number of subdomains with $\frac{H}{h}=8$ and $\tau_{\kappa}=h_{\kappa}$. 
TABLE 7.3

Performance of solving (4.2) with the HDG discretization $\left(\tau_{\kappa}=1 / h_{\kappa}\right)$.

\begin{tabular}{|c|c|c|c|c|c|c|c|}
\hline \multirow[b]{2}{*}{$H / h$} & \multirow[b]{2}{*}{ \#sub } & \multicolumn{2}{|c|}{$k=0$} & \multicolumn{2}{|c|}{$k=1$} & \multicolumn{2}{|c|}{$k=2$} \\
\hline & & Cond. & Iter. & Cond. & Iter. & Cond. & Iter. \\
\hline \multirow[t]{5}{*}{8} & $4 \times 4$ & 4.21 & 10 & 4.72 & 12 & 12.72 & 14 \\
\hline & $8 \times 8$ & 5.12 & 12 & 8.81 & 17 & 11.52 & 20 \\
\hline & $16 \times 16$ & 5.00 & 13 & 10.43 & 21 & 13.44 & 24 \\
\hline & $24 \times 24$ & 5.14 & 13 & 10.83 & 20 & 13.96 & 25 \\
\hline & $32 \times 32$ & 5.14 & 13 & 10.84 & 20 & 14.09 & 25 \\
\hline \#sub & $H / h$ & Cond. & Iter. & Cond. & Iter. & Cond. & Iter. \\
\hline \multirow[t]{5}{*}{$8 \times 8$} & 4 & 2.56 & 9 & 6.23 & 14 & 8.52 & 17 \\
\hline & 8 & 5.12 & 12 & 8.81 & 17 & 11.52 & 20 \\
\hline & 16 & 7.59 & 15 & 11.86 & 20 & 17.86 & 24 \\
\hline & 24 & 9.22 & 17 & 13.86 & 22 & 20.32 & 25 \\
\hline & 32 & 10.48 & 19 & 15.37 & 23 & 22.21 & 26 \\
\hline
\end{tabular}

Acknowledgments. The authors thank the referees for carefully reading our manuscript and giving useful suggestions. The first and third authors' work were supported in part by the NSF under Contract DMS-1723066.

\section{REFERENCES}

[1] L. Beirão Da Veiga, D. Cho, L. F. PaVARIno, AND S. SCACChI, BDDC preconditioners for isogeometric analysis, Math. Models Methods Appl. Sci., 23 (2013), pp. 1099-1142.

[2] L. Beirão da Veiga, L. F. Pavarino, S. Scacchi, O. B. Widlund, and S. Zampini, Isogeometric BDDC preconditioners with deluxe scaling, SIAM J. Sci. Comput., 36 (2014), pp. A1118-A1139.

[3] J. Bramble And J. Pasciak, A domain decomposition technique for Stokes problems, Appl. Numer. Math., 6 (1990), pp. 251-261.

[4] C. CANUto, L. F. PAVARINO, AND A. B. Pieri, BDDC preconditioners for continuous and discontinuous Galerkin methods using spectral/hp elements with variable local polynomial degree, IMA J. Numer. Anal., 34 (2014), pp. 879-903.

[5] B. Cockburn, O. Dubois, J. Gopalakrishnan, and S. Tan, Multigrid for an HDG method, IMA J. Numer. Anal., 34 (2014), pp. 1386-1425.

[6] B. Cockburn, J. Gopalakrishnan, N. C. Nguyen, J. Peraire, and F. Sayas, Analysis of HDG methods for Stokes flow, Math. Comp., 80 (2011), pp. 723-760.

[7] C. R. Dohrmann, A preconditioner for substructuring based on constrained energy minimization, SIAM J. Sci. Comput., 25 (2003), pp. 246-258.

[8] — - An approximate BDDC preconditioner, Numer. Linear Algebra Appl., 14 (2007), pp. 149-168.

[9] - Preconditioning of saddle point systems by substructuring and a penalty approach, in Domain Decomposition Methods in Science and Engineering XVI, O. B. Widlund and D. E. Keyes, eds., Lect. Notes Comput. Sci. Eng., vol. 55, Springer, Berlin, 2007, pp. 53-64.

[10] P. GoldFeld, L. PaVARINO, AND O. WidLund, Balancing Neumann-Neumann preconditioners for mixed approximations of heterogeneous problems in linear elasticity, Numer. Math., 95 (2003), pp. 283-324.

[11] J. Gopalakrishnan, A Schwarz preconditioner for a hybridized mixed method, Comput. Methods Appl. Math., 3 (2003), pp. 116-134.

[12] H. H. KIM, E. T. CHUNG, AND C. S. LEE, A BDDC algorithm for a class of staggered discontinuous Galerkin methods, Comput. Math. Appl., 67 (2014), pp. 1373-1389.

[13] H. H. KIM AND X. TU, A three-level BDDC algorithm for mortar discretization, SIAM J. Numer. Anal., 47 (2009), pp. 1576-1600.

[14] A. KLAWOnN And O. Rheinbach, Inexact FETI-DP methods, Internat. J. Numer. Methods Engrg., 69 (2007), pp. 284-307.

[15] A. Klawonn AND O. B. Widlund, Dual-primal FETI methods for linear elasticity, Comm. Pure Appl. Math., 59 (2006), pp. 1523-1572. 
[16] J. LI, A Dual-Primal FETI method for incompressible Stokes equations, Numer. Math., 102 (2005), pp. 257275.

[17] J. LI AND X. TU, Convergence analysis of a balancing domain decomposition method for solving interior Helmholtz equations, Numer. Linear Algebra Appl., 16 (2009), pp. 745-773.

[18] - A nonoverlapping domain decomposition method for incompressible Stokes equations with continuous pressures, SIAM J. Numer. Anal., 51 (2013), pp. 1235-1253.

[19] — A FETI-DP algorithm for incompressible Stokes equations with continuous pressures, in Domain Decomposition Methods in Science and Engineering XXI, J. Erhel, M. J. Gander, L. Halpern, G. Pichot, T. Sassi, and O. Widlund, eds., Lect. Notes Comput. Sci. Eng., vol. 98, Springer, Berlin, 2014, pp. 157-165.

[20] J. LI AND O. WIDLUND, BDDC algorithms for incompressible Stokes equations, SIAM J. Numer. Anal., 44 (2006), pp. 2432-2455.

[21] — - FETI-DP, BDDC, and block Cholesky methods, Internat. J. Numer. Methods Engrg., 66 (2006), pp. 250-271.

[22] J. MANDEL AND C. R. Dohrmann, Convergence of a balancing domain decomposition by constraints and energy minimization, Numer. Linear Algebra Appl., 10 (2003), pp. 639-659.

[23] J. Mandel, C. R. Dohrmann, AND R. Tezaur, An algebraic theory for primal and dual substructuring methods by constraints, Appl. Numer. Math., 54 (2005), pp. 167-193.

[24] N. C. NguYen, J. Peraire, AND B. Cockburn, A hybridizable discontinuous Galerkin method for Stokes flow, Comput. Methods Appl. Mech. Engrg., 199 (2010), pp. 582-597.

[25] L. PAVARINO AND S. SCACCHI, Isogeometric block FETI-DP preconditioners for the Stokes and mixed linear elasticity systems, Comput. Methods Appl. Mech. Engrg., 310 (2016), pp. 694-710.

[26] L. PaVARINO AND O. WidLund, Balancing Neumann-Neumann methods for incompressible Stokes equations, Comm. Pure Appl. Math., 55 (2002), pp. 302-335.

[27] L. PAVARINO, O. WIDLUND, AND S. ZAMPINI, BDDC preconditioners for spectral element discretizations of almost incompressible elasticity in three dimensions, SIAM J. Sci. Comput., 32 (2010), pp. 3604-3626.

[28] X. TU, A BDDC algorithm for a mixed formulation of flows in porous media, Electron. Trans. Numer. Anal., 20 (2005), pp. 164-179.

http://etna.ricam.oeaw.ac.at/vol.20.2005/pp164-179.dir/pp164-179.pdf

[29] - A BDDC algorithm for flow in porous media with a hybrid finite element discretization, Electron. Trans. Numer. Anal., 26 (2007), pp. 146-160.

http://etna.ricam.oeaw.ac.at/vol.26.2007/pp146-160.dir/pp146-160.pdf

[30] - Three-level BDDC, in Domain Decomposition Methods in Science and Engineering XVI, O. B. Widlund and D. E. Keyes, eds., Lect. Notes Comput. Sci. Eng., vol. 55, Springer, Berlin, 2007, pp. 437-444.

[31] - Three-level BDDC in three dimensions, SIAM J. Sci. Comput., 29 (2007), pp. 1759-1780.

[32] - Three-level BDDC in two dimensions, Internat. J. Numer. Methods Engrg., 69 (2007), pp. 33-59.

[33] —, A three-level BDDC algorithm for saddle point problems, Numer. Math., 119 (2011), pp. 189-217.

[34] X. TU AND J. LI, A balancing domain decomposition method by constraints for advection-diffusion problems, Commun. Appl. Math. Comput. Sci., 3 (2008), pp. 25-60.

[35] _ - A unified dual-primal finite element tearing and interconnecting approach for incompressible Stokes equations, Internat. J. Numer. Methods Engrg., 94 (2013), pp. 128-149.

[36] - A FETI-DP type domain decomposition algorithm for three-dimensional incompressible Stokes equations, SIAM J. Numer. Anal., 53 (2015), pp. 720-742.

[37] X. TU AND B. WANG, A BDDC algorithm for second-order elliptic problems with hybridizable discontinuous Galerkin discretizations, Electron. Trans. Numer. Anal., 45 (2016), pp. 354-370. http://etna.ricam.oeaw.ac.at/vol.45.2016/pp354-370.dir/pp354-370.pdf

[38] _ A BDDC algorithm for the Stokes problem with weak Galerkin discretizations, Comput. Math. Appl., 76 (2018), pp. 377-392.

[39] J. WANG AND X. YE, A weak Galerkin finite element method for the Stokes equations, Adv. Comput. Math., 42 (2016), pp. 155-174.

[40] S. ZAMPINI AND X. TU, Multilevel balancing domain decomposition by constraints deluxe algorithms with adaptive coarse spaces for flow in porous media, SIAM J. Sci. Comput., 39 (2017), pp. A1389-A1415. 